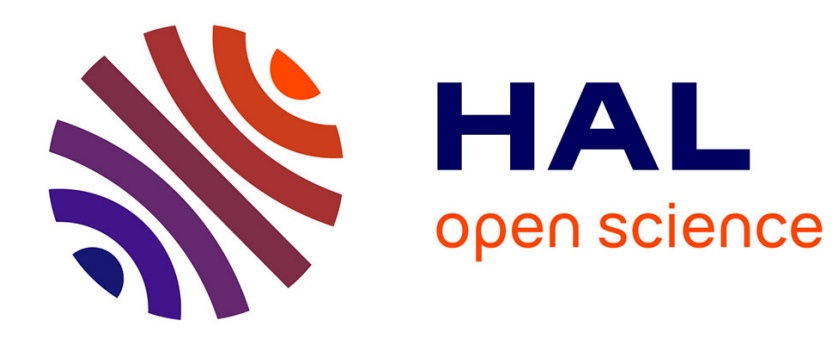

\title{
Very Regular Solutions for the Landau-Lifschitz Equation with Electric Current
}

Gilles Carbou, Rida Jizzini

\section{To cite this version:}

Gilles Carbou, Rida Jizzini. Very Regular Solutions for the Landau-Lifschitz Equation with Electric Current. Chinese Annals of Mathematics - Series B, 2018, 39 (5), pp.889-916. hal-01725842

\section{HAL Id: hal-01725842 \\ https://hal.science/hal-01725842}

Submitted on 7 Mar 2018

HAL is a multi-disciplinary open access archive for the deposit and dissemination of scientific research documents, whether they are published or not. The documents may come from teaching and research institutions in France or abroad, or from public or private research centers.
L'archive ouverte pluridisciplinaire HAL, est destinée au dépôt et à la diffusion de documents scientifiques de niveau recherche, publiés ou non, émanant des établissements d'enseignement et de recherche français ou étrangers, des laboratoires publics ou privés. 


\title{
Very Regular Solutions for the Landau-Lifschitz Equation with Electric Current
}

\author{
Gilles CARBOU ${ }^{1}$ AND RidA JIZZINI ${ }^{2}$ \\ ${ }^{1}$ Laboratoire de Mathématiques Appliquées de Pau, UMR CNRS 5142, Université de Pau et des Pays de l'Adour, \\ Avenue de l'Université - BP 1155, 64013 Pau, Cedex France. \\ Email: gilles.carbou@univ-pau.fr \\ 2 Institut de Mathématiques de Bordeaux, UMR CNRS 5251, Université de Bordeaux, 351 cours de la Libération, \\ 33405 Talence cedex, France. \\ Email: Rida.Jizzini@math.u-bordeaux1.fr
}

\begin{abstract}
We consider a model of ferromagnetic material subject to an electric current. We prove the local in time existence of very regular solutions for this model in the scale of $H^{k}$ spaces. In particular, we describe in detail the compatibility conditions at the boundary for the initial data.
\end{abstract}

Keywords: ferromagnetic materials, compatibility conditions, Existence result

\section{Introduction}

Ferromagnetic materials are used in several applications: radar furtivity, electromagnets, storage of digital data. They are characterized by a spontaneous magnetization represented by the magnetic moment $m$ defined on $[0, T] \times \Omega$, where $\Omega$ is the ferromagnetic domain in which the material is confined. At low temperature (under the Curie temperature), the material is said to be saturated, that is the norm of the magnetic moment is constant, so after renormalization, we have:

$$
\forall t \in[0, T], \quad \forall x \in \Omega, \quad|m(t, x)|=1 .
$$

The magnetic moment links the magnetic field $H$ and the magnetic induction $B$ by the constitutive relation:

$$
B=H+\bar{m},
$$

where $\bar{m}$ is the extension of $m$ by zero outside $\Omega$.

The most promising application of the ferromagnetic materials concerns the nano-electronics, and in particular the storage of digital informations with quick access. The ferromagnetic devices used for these applications are either nano wires, thin plates or really 3d components. For nano-electronic applications, one goal is to store informations by magnetic domains representing the bits, and transmit it very rapidly along the magnetic structures via domain walls motion. A standard way to induce this motion is by applying a magnetic field on the sample. The effects of the applied magnetic field are measured by the Zeeman energy (see $[5,11,15]$ ). The problem of this solution is that the information is modified since the domains can be removed by the application of an applied field. As explained in $[13,14]$, the good way to transport the information (to a reading head for example) without change is obtained by using of an applied electric current. This increases the access velocity to the memory without mechanical wear of the components, and preserving the data.

In this paper, we study the following Landau-Lifschitz model describing a ferromagnetic material submitted to an applied current: 


$$
\begin{cases}\frac{\partial m}{\partial t}=-m \times H_{e}(m)-m \times\left(m \times H_{e}(m)\right)+(v \cdot \nabla) m+m \times(v \cdot \nabla) m & \text { in }[0, T] \times \Omega, \\ H_{e}(m)=\Delta m+H(m), & \text { on }[0, T] \times \partial \Omega, \\ \frac{\partial m}{\partial \nu}=0 & \text { in } \Omega, \\ m(0, x)=m_{0}(x) & \end{cases}
$$

where:

- $\Omega$ is an open bounded set with smooth boundary and $\nu$ is the outside unit normal on $\partial \Omega$,

- $H_{e}$ is the effective magnetic field including the demagnetizing field and the exchange field,

- $v$ is the current speed. For physical reasons, the relevant boundary condition is that $v$ satisfies $v \cdot \nu=0$ on the boundary (the electric current remains in the domain), but we do not use this condition in our analysis.

- $\Delta m$ is the exchange field,

- $H(m)$ is the demagnetizing field. It is deduced from $m$ by the static Maxwell equations and the law of Faraday:

$$
\left\{\begin{array}{l}
\operatorname{curl} H(m)=0 \text { in } \mathbb{R}^{3}, \\
\operatorname{div}(H(m)+\bar{m})=0 \text { in } \mathbb{R}^{3},
\end{array}\right.
$$

where $\bar{m}$ is the extension of $m$ by zero outside $\Omega$.

Remark 1. The modelisation of the applied current by the transport term $(v \cdot \nabla) m+m \times(v \cdot \nabla) m$ is explained in [13] (see also the references therein).

We denote by $H^{k}\left(\Omega ; S^{2}\right)$ the set:

$$
H^{k}\left(\Omega ; S^{2}\right)=\left\{u: \Omega \rightarrow \mathbb{R}^{3}, u \in H^{k}(\Omega) \text { and }|u(x)|=1 \text { a.e. }\right\} .
$$

In [1], [6] and [16], the existence of global weak solutions for the Landau-Lifschitz equation without electric current in obtained. With the same method as [1] or [6], G. Bonithon proves in [3] the global existence of weak solutions for (2). In the case of the Landau-Lifchitz equations without electric current when the effective field is reduced to $\Delta m$, the weak solutions are non unique (see [1]). This non uniqueness is expected in more general cases but is totally open. In addition, the Landau-Lifschitz equation does not have regularizing effects. So the existence of strong solutions is not obvious.

Existence of strong solutions for the Landau-Lifschitz equation without applied current is obtained in [7] and [8]. The solutions constructed in these papers are in $L^{\infty}\left(0, T ; H^{2}(\Omega)\right) \cap L^{2}\left(0, T ; H^{3}(\Omega)\right)$ and are local in time.

In the present paper, following the method of [7], we construct local in time strong solutions for (2) in $L^{\infty}\left(0, T^{*} ; H^{2}(\Omega)\right) \cap L^{2}\left(0, T^{*} ; H^{3}(\Omega)\right)$. In addition, for more regular initial data, if compatibility conditions are satisfied, we prove the existence of strong solutions with high regularity on the same maximal existence interval $\left[0, T^{*}[\right.$. In particular, we prove that an eventual blow up for the regular solution occurs for the $H^{2}$ norm. To our knowledge, the construction of very regular solutions for the Landau-Lifschitz equation does not exist in the literature, even for models without applied current. Our first result is the following: 
Theorem 1.1. Let $m_{0} \in H^{2}\left(\Omega ; S^{2}\right)$ satisfying the compatibility condition:

$$
\frac{\partial m_{0}}{\partial \nu}=0 \text { on } \partial \Omega
$$

Let $v \in \mathcal{C}^{0}\left(\mathbb{R}^{+} ; L^{\infty}(\Omega) \cap W^{1,3}(\Omega)\right)$. There exist a time $T^{*}>0$ depending only on the size of the initial data and a unique $m$ such that for all $T<T^{*}$,

- $m \in \mathcal{C}^{0}\left(0, T ; H^{2}(\Omega)\right) \cap L^{2}\left(0, T ; H^{3}(\Omega)\right)$.

- $|m(t, x)|=1 \quad$ in $\quad[0, T] \times \Omega$.

- $m$ satisfies (2).

Without additional compatibility condition, we can construct solutions in $H^{3}(\Omega)$ :

Theorem 1.2. Let $m_{0} \in H^{3}\left(\Omega, S^{2}\right)$ such that $\frac{\partial m_{0}}{\partial \nu}=0$ on $\partial \Omega$. Let $v \in \mathcal{C}^{0}\left(\mathbb{R}^{+} ; L^{\infty}(\Omega) \cap\right.$ $\left.W^{1,3}(\Omega)\right) \cap \in \mathcal{C}^{1}\left(\mathbb{R}^{+} ; L^{2}(\Omega)\right)$. Let $T^{*}>0$ and $m \in L^{\infty}\left(0, T ; H^{2}(\Omega)\right) \cap L^{2}\left(0, T ; H^{3}(\Omega)\right)$ for $T<T^{*}$ given by Theorem 1.1. Then, for all $T<T^{*}$ :

$$
m \in \mathcal{C}^{0}\left(0, T ; H^{3}(\Omega)\right) \cap L^{2}\left(0, T ; H^{4}(\Omega)\right) .
$$

The key point for the proof of Theorems 1.1 and 1.2 is that the Landau-Lifschitz equation (2) is equivalent, for solutions satisfying the saturation constraint (1), to the following problem:

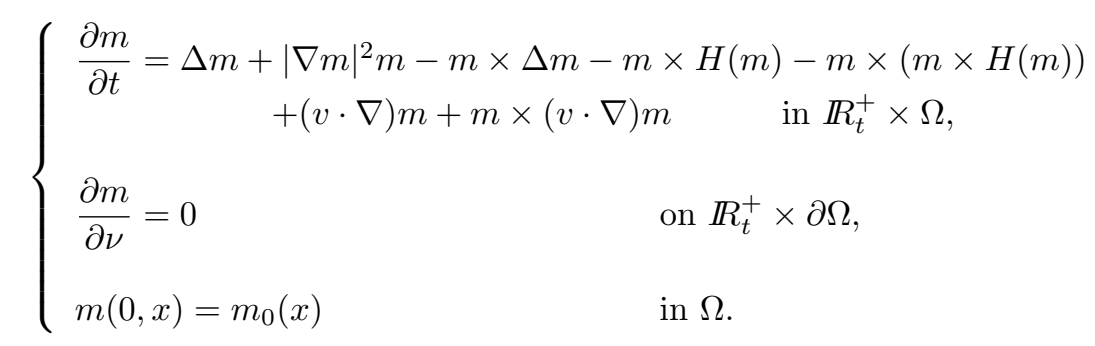

In order to obtain more regularity for the solutions of (4), we must assume compatibility conditions on the initial data. Since (2) is equivalent to (4), and since our strategy consists in working with this last equation, we write the compatibility conditions derived from (4). First, we define formally the initial value of $\partial_{t}^{k} m$ at $t=0$.

We set $V_{0}=m_{0}$. We denote $v_{i}(x):=\partial_{t}^{i} v(0, x)$.

Using equation (4), if $m$ is regular enough, we obtain the value of $\partial_{t} m_{\mid t=0}$ by taking (4) at $t=0$ and by writing that $m_{\mid t=0}=m_{0}$. So, we define $V_{1}$ by:

$$
\begin{aligned}
V_{1} & =\Delta m_{0}+\left|\nabla m_{0}\right|^{2} m_{0}-m_{0} \times\left(\Delta m_{0}+H\left(m_{0}\right)\right)-m_{0} \times\left(m_{0} \times\left(H\left(m_{0}\right)\right)\right) \\
& +\left(v_{0} \cdot \nabla\right) m_{0}+m_{0} \times\left(v_{0} \cdot \nabla\right) m_{0} .
\end{aligned}
$$

We remark that, if $m_{0} \in H^{k}(\Omega)$ and if $v$ is sufficiently regular, then $V_{1} \in H^{k-2}(\Omega)$ for $k \geq 2$.

In order to write the compatibility conditions, we recall the following formulas for the derivation of a product:

$$
\partial_{t}^{K}(a b)=\sum_{i=0}^{K} C_{K}^{i} \partial_{t}^{i} a \partial_{t}^{K-i} b, \quad \text { with } C_{K}^{i}=\frac{k !}{(k-i) ! i !},
$$

and

$$
\partial_{t}^{K}(a b c)=\sum_{\alpha \in A_{K}} C^{\alpha} \partial_{t}^{\alpha_{1}} a \partial_{t}^{\alpha_{2}} b \partial_{t}^{\alpha_{3}} c
$$

with:

$$
\text { - } A_{K}=\left\{\alpha=\left(\alpha_{1}, \alpha_{2}, \alpha_{3}\right) \in\{0, \ldots, K\}^{3}, \quad \alpha_{1}+\alpha_{2}+\alpha_{3}=K\right\},
$$




$$
\text { - } C^{\alpha}=C_{\alpha_{1}+\alpha_{2}+\alpha_{3}}^{\alpha_{1}+\alpha_{2}} \cdot C_{\alpha_{1}+\alpha_{2}}^{\alpha_{1}} \text {. }
$$

By differentiating (4) $k$ times with respect to $t$, and by taking the obtained result at $t=0$, we define recursively $V_{k+1}: \Omega \longrightarrow \mathbb{R}^{3}$ by:

$$
\begin{aligned}
V_{k+1}= & \Delta V_{k}+\sum_{\alpha \in A_{k}} C^{\alpha}\left(\nabla V_{\alpha_{1}} \cdot \nabla V_{\alpha_{2}}\right) V_{\alpha_{3}}-\sum_{j=0}^{k} C_{k}^{j} V_{j} \times\left(\Delta V_{k-j}+H\left(V_{k-j}\right)\right) \\
& -\sum_{\alpha \in A_{k}} C^{\alpha}\left(V_{\alpha_{1}} \times\left(V_{\alpha_{2}} \times H\left(V_{\alpha_{3}}\right)\right)-V_{\alpha_{1}} \times\left(v_{\alpha_{2}} \cdot \nabla\right) V_{\alpha_{3}}\right)+\sum_{j=0}^{k} C_{k}^{j}\left(v_{j} \cdot \nabla\right) V_{k-j} .
\end{aligned}
$$

We remark that if $m_{0} \in H^{2 k+2+p}(\Omega)$, then $V_{k+1} \in H^{p}(\Omega)$.

Definition 1.3. Let $k \in \mathbb{N}$, let $m_{0} \in H^{2 k+2}\left(\Omega ; S^{2}\right)$. We say that $m_{0}$ satisfies the compatibility condition at order $k$ if :

$$
\forall i \in\{0,1, \ldots, k\}, \quad \frac{\partial V_{i}}{\partial \nu}=0 \text { on } \partial \Omega .
$$

Theorem 1.4. Let $k \geq 4$. Let $m_{0} \in H^{k}\left(\Omega ; S^{2}\right)$ and let $m$ and $T^{*}$ given in Theorem 1.1. We assume that $m_{0}$ satisfies the compatibility condition at order $\left[\frac{k}{2}\right]-1$. In addition, we assume that

$$
\forall j \leq k-\left[\frac{k}{2}\right]-1, \quad \partial_{t}^{i} v \in \mathcal{C}^{0}\left(0, T ; H^{k-2 j}(\Omega)\right) \cap L^{2}\left(0, T ; H^{k-2 j+1}(\Omega)\right) .
$$

Then, for all $T<T^{*}$,

$$
m \in L^{\infty}\left(0, T ; H^{k}(\Omega)\right) \cap L^{2}\left(0, T ; H^{k+1}(\Omega)\right),
$$

and

$$
\forall j \in 1, \ldots,\left[\frac{k}{2}\right]-1, \quad \frac{\partial^{j} m}{\partial t^{j}} \in L^{\infty}\left(0, T ; H^{k-2 j}(\Omega)\right) \cap L^{2}\left(0, T ; H^{k-2 j+1}(\Omega)\right) .
$$

The present paper is organized as follows.

In Section 2, we recall useful lemmas. In particular, we study the regularity properties for the operator $H$.

Section 3 is devoted to the proof of Theorem 1.1. Basically, we follow the method of [7]. As we said before, the key point is that Equation (2) is equivalent to (4) for solutions satisfying the saturation constraint (1). In this new formulation, the dissipation due to the exchange field $\Delta m$ appears completely though it only appears $m \times \Delta m$ in (2). We construct by the Galerkin method a solution of (4) in $L^{\infty}\left(0, T ; H^{2}(\Omega)\right) \cap L^{2}\left(0, T ; H^{3}(\Omega)\right)$, and we prove that this solution satisfies in addition the saturation constraint, so that it is a solution for (2).

Construction of more regular solutions for (2) is totally new and entails several difficulties. In Section 4, in order to prove the $H^{3}$ regularity for the solution of (4), a direct energy estimate for the third order space derivatives is not possible because of the non local term $H(m)$ which does not satisfy the homogeneous Neumann boundary condition. Our method consists in differentiating the Galerkin approximation with respect to time and we prove by this way that $\partial_{t} m \in L^{\infty}\left(0, T ; H^{1}(\Omega)\right) \cap$ $L^{2}\left(0, T ; H^{2}(\Omega)\right)$. We recover the $H^{3}$-regularity for $m$ by a bootstrap argument using Equation (4). This operation is complicated by the non linear term $|\nabla m|^{2} m$.

Section 5 is devoted to the proof of Theorem 1.4. As already said for the proof of the $H^{3}$-regularity, variational estimates for high order space derivatives are not possible because of the non local term $H(m)$ which does not satisfy the homogeneous Neumann boundary condition. In addition, we are unable to perform $H^{2}$ estimates for the time derivative of $m_{n}$, solution for the Galerkin approximation of (4). Indeed, we need for that compatibility conditions for all $n$ which are not satisfied because of the non local term $H\left(m_{n}\right)$.

Let us explain briefly our strategy in the simplest case: $k=4$. We already know from Theorem 1.2 that the solution $m$ is in $L^{\infty}\left(0, T ; H^{3}(\Omega)\right) \cap L^{2}\left(0, T ; H^{4}(\Omega)\right)$. We derivate (4) with respect to time. 
By a Galerkin process, using compatibility conditions on $\partial_{t} m(t=0)$, we construct a solution for the obtained problem in $L^{\infty}\left(0, T ; H^{2}(\Omega)\right) \cap L^{2}\left(0, T ; H^{3}(\Omega)\right)$. Now, $\partial_{t} m$ is a solution of this problem and a uniqueness argument ensures then that $\partial_{t} m \in L^{\infty}\left(0, T ; H^{2}(\Omega)\right) \cap L^{2}\left(0, T ; H^{3}(\Omega)\right)$. Finally, we improve the regularity of $m$ by elliptic regularity theorems writing $\Delta m$ in function of $\partial_{t} m$.

In the general case, we proceed by induction with, roughly speaking, the same method.

\section{Preliminary results}

\subsection{Equivalent norms}

We use the following notations:

$$
\|u\|_{2, \Omega}=\left(\|u\|_{L^{2}}^{2}+\|\Delta u\|_{L^{2}}^{2}\right)^{\frac{1}{2}},
$$

and

$$
\|\nabla u\|_{1, \Omega}=\left(\|\nabla u\|_{L^{2}}^{2}+\|\Delta u\|_{L^{2}}^{2}\right)^{\frac{1}{2}} .
$$

We recall the following result established in [7]:

Lemma 2.1. Let $\Omega$ be a bounded regular open set. There exist $c_{1}$ and $c_{2}$ such that for all $u \in H^{2}(\Omega)$ such that $\frac{\partial u}{\partial \nu}=0$ on $\partial \Omega$,

$$
\begin{gathered}
c_{1}\|u\|_{2, \Omega} \leq\|u\|_{H^{2}(\Omega)} \leq c_{2}\|u\|_{2, \Omega}, \\
c_{1}\|\nabla u\|_{1, \Omega} \leq\|\nabla u\|_{H^{1}(\Omega)} \leq c_{2}\|\nabla u\|_{1, \Omega},
\end{gathered}
$$

and for all $u \in H^{3}(\Omega)$ such that $\frac{\partial u}{\partial \nu}=0$ on $\partial \Omega$,

$$
c_{1}\left(\|\nabla u\|_{1, \Omega}^{2}+\|\nabla \Delta u\|_{L^{2}}^{2}\right)^{\frac{1}{2}} \leq\|\nabla u\|_{H^{2}(\Omega)} \leq c_{2}\left(\|\nabla u\|_{1, \Omega}^{2}+\|\nabla \Delta u\|_{L^{2}}^{2}\right)^{\frac{1}{2}} .
$$

From Lemma 2.1 and using the standard interpolation inequality, we rewrite Sobolev and GagliardoNirenberg inequalities on the form

Lemma 2.2. Let $\Omega$ be a regular bounded domain of $\mathbb{R}^{3}$. There exists a constant $C$ such that for all $u \in H^{2}(\Omega)$ such that $\frac{\partial u}{\partial \nu}=0$ on $\partial \Omega$,

$$
\begin{gathered}
\|u\|_{L^{\infty}} \leq C\|u\|_{2, \Omega}, \\
\|\nabla u\|_{L^{6}} \leq C\|u\|_{2, \Omega}, \\
\|\nabla u\|_{L^{4}}^{2} \leq C\|u\|_{L^{\infty}}\|u\|_{2, \Omega},
\end{gathered}
$$

and for all $u \in H^{3}(\Omega)$ such that $\frac{\partial u}{\partial \nu}=0$ on $\partial \Omega$,

$$
\left\|D^{2} u\right\|_{L^{3}} \leq C\left(\|u\|_{2, \Omega}+\|u\|_{2, \Omega}^{\frac{1}{2}}\|\nabla \Delta u\|_{L^{2}}^{\frac{1}{2}}\right) .
$$

The following lemma will be useful to estimate products of functions.

Lemma 2.3. If $u \in H^{1}(\Omega)$ and $v \in H^{2}(\Omega)$, then $u v \in H^{1}(\Omega)$ and

$$
\|u v\|_{H^{1}(\Omega)} \leq C\|u\|_{H^{1}(\Omega)}\|v\|_{H^{2}(\Omega)}
$$

Proof. Since $\Omega$ is a smooth bounded domain of $\mathbb{R}^{3}$, by Sobolev embedding, $H^{2}(\Omega) \subset L^{\infty}(\Omega)$ so that $u v \in L^{2}(\Omega)$.

In addition, $\nabla(u v)=(\nabla u) v+u \nabla v$. By the same argument, $(\nabla u) v \in L^{2}(\Omega)$. Since $H^{1}(\Omega) \subset L^{4}(\Omega)$, then $u \nabla v \in L^{2}(\Omega)$. 


\subsection{Comparison lemma}

We recall without proof the following standard comparison lemma:

Lemma 2.4. Let $f: \mathbb{R}^{+} \times \mathbb{R} \longrightarrow \mathbb{R}, \mathcal{C}^{0}$ and locally lipschtz with respect to its second variable. Let $z:\left[0, T^{*}[\rightarrow \mathbb{R}\right.$ be the maximal solution of the Cauchy problem:

$$
\left\{\begin{array}{l}
z^{\prime}=f(t, z) \\
z(0)=z_{0}
\end{array}\right.
$$

Let $y: \mathbb{R}^{+} \rightarrow \mathbb{R}, \mathcal{C}^{1}$ such that

$$
\left\{\begin{array}{l}
\forall t \geq 0, \quad y^{\prime}(t) \leq f(t, y(t)) \\
y(0) \leq z_{0}
\end{array}\right.
$$

Then

$$
\forall t \in\left[0, T^{*}[, \quad y(t) \leq z(t) .\right.
$$

\subsection{Galerkin basis}

Let us denote by $V_{n}$ the finite space spanned on the $n$ first eigenfunctions of the operator $A=-\Delta+I$ with $D(A)=\left\{u \in H^{2}(\Omega)\right.$ such that $\frac{\partial u}{\partial \nu}=0$ on $\left.\partial \Omega\right\}$, and $P_{n}$, the orthogonal projection from $L^{2}(\Omega)$ onto $V_{n}$.

Proposition 1. There exists a constant $C$ such that for all $n$, the orthogonal projection $P_{n}$ satisfies the following properties:

- For all $u \in H^{1}(\Omega), \quad\left\|P_{n}(u)\right\|_{H^{1}(\Omega)} \leq\|u\|_{H^{1}(\Omega)}$,

- For all $u \in H^{2}(\Omega)$ such that $\frac{\partial u}{\partial \nu}=0, \quad\left\|P_{n}(u)\right\|_{H^{2}(\Omega)} \leq C\|u\|_{H^{2}(\Omega)}$,

- For all $u \in H^{3}(\Omega)$ such that $\frac{\partial u}{\partial \nu}=0, \quad\left\|P_{n}(u)\right\|_{H^{3}(\Omega)} \leq C\|u\|_{H^{3}(\Omega)}$.

Proof. We write $u$ on the form $u=P_{n}(u)+Q_{n}(u)$, where $Q_{n}(u)$ belongs to $V_{n}^{\perp}$. Since $\|u\|_{L^{2}}^{2}=$ $\left\|P_{n}(u)\right\|_{L^{2}}^{2}+\left\|Q_{n}(u)\right\|_{L^{2}}^{2}$, we obtain

$$
\left\|P_{n}(u)\right\|_{L^{2}} \leq\|u\|_{L^{2}} .
$$

Using integration by parts and the fact that $\Delta P_{n}(u)$ belongs to $V_{n}$ so that $\frac{\partial P_{n}(u)}{\partial \nu}=0$ on $\partial \Omega$, we obtain

$$
\begin{aligned}
\left\|\nabla P_{n}(u)\right\|_{L^{2}}^{2} & =-\int_{\Omega} \Delta P_{n}(u) \cdot P_{n}(u) d x \\
& =-\int_{\Omega} \Delta P_{n}(u) \cdot u d x \quad \text { (since } \Delta P_{n}(u) \text { and } Q_{n}(u) \text { are orthogonal) } \\
& \left.=\int_{\Omega} \nabla P_{n}(u) \cdot \nabla u d x \quad \text { (since } P_{n}(u) \in V_{n}\right) \\
& \leq\left\|\nabla P_{n}(u)\right\|_{L^{2}}\|\nabla u\|_{L^{2}} .
\end{aligned}
$$

So

$$
\left\|\nabla P_{n}(u)\right\|_{L^{2}} \leq\|\nabla u\|_{L^{2}}
$$


In the same way, for the $H^{2}$ and the $H^{3}$ estimates, we remark that $\frac{\partial \Delta P_{n}(u)}{\partial \nu}=\frac{\partial \Delta^{2} P_{n}(u)}{\partial \nu}=0$ on the boundary. For the $H^{2}$ estimate, we have:

$$
\begin{array}{rlr}
\left\|\Delta P_{n}(u)\right\|_{L^{2}}^{2} & =-\int_{\Omega} \nabla \Delta P_{n}(u) \cdot \nabla P_{n}(u) d x & \\
& =\int_{\Omega} \Delta^{2} P_{n}(u) \cdot P_{n}(u) d x & \\
& =\int_{\Omega} \Delta^{2} P_{n}(u) \cdot u d x & \\
& =-\int_{\Omega} \nabla \Delta P_{n}(u) \cdot \nabla u d x & \\
& & \\
& =\int_{\Omega} \Delta P_{n}(u) \cdot \Delta u d x & \\
& \leq\left\|\Delta P_{n}(u)\right\|_{L^{2}}\|\Delta u\|_{L^{2}} . &
\end{array}
$$

So, $\left\|\Delta P_{n}(u)\right\|_{L^{2}} \leq\|\Delta u\|_{L^{2}}$ and therefore:

$$
\left\|P_{n}(u)\right\|_{H^{2}} \leq C\|u\|_{H^{2}} .
$$

For the $H^{3}$ estimate,

$$
\begin{aligned}
& \left\|\nabla \Delta P_{n}(u)\right\|_{L^{2}}^{2}=-\int_{\Omega} \Delta^{2} P_{n}(u) \cdot \Delta P_{n}(u) d x \\
& =\int_{\Omega} \nabla \Delta^{2} P_{n}(u) \cdot \nabla P_{n}(u) d x \\
& =-\int_{\Omega} \Delta^{3} P_{n}(u) \cdot P_{n}(u) d x \\
& =-\int_{\Omega} \Delta^{3} P_{n}(u) \cdot u d x \quad\left(\text { since } \Delta^{3} P_{n}(u) \in V_{n}\right) \\
& =\int_{\Omega} \nabla \Delta^{2} P_{n}(u) \cdot \nabla u d x \\
& \left.=-\int_{\Omega} \Delta^{2} P_{n}(u) \cdot \Delta u d x \quad \text { (using that } \frac{\partial u}{\partial \nu}=0 \text { on } \partial \Omega\right) \text {, } \\
& =\int_{\Omega} \nabla \Delta P_{n}(u) \cdot \nabla \Delta u d x \\
& \leq\left\|\nabla \Delta P_{n}(u)\right\|_{L^{2}}\|\nabla \Delta u\|_{L^{2}} .
\end{aligned}
$$

Therefore, using Lemma 2.1,

$$
\left\|P_{n}(u)\right\|_{H^{3}} \leq\|u\|_{H^{3}} .
$$

\subsection{Demagnetizing field}

The operator $H$ takes its values in the space $L^{2}\left(\mathbb{R}^{3}\right)$. We can observe that $u \longrightarrow-H(u)$ is the orthogonal projection of $\bar{u}$ on the vector fields of gradients in $L^{2}\left(\mathbb{R}^{3}\right)$. Let us consider the restriction 
of $H$ to $\Omega$. Classicaly, we have

$$
\|H(u)\|_{L^{p}(\Omega)} \leq C\|u\|_{L^{p}(\Omega)} \quad \text { for } 1<p<+\infty .
$$

In the proposition below, following Ladyshenskaya [10] page 196 we can derive the following regularity result which describe the continuity of the operator $H$ on the spaces $W^{k, p}(\Omega)$ for $\left.p \in\right] 1,+\infty[$ and $k \in \mathbb{N}$.

Proposition 2. Let $p \in] 1,+\infty\left[\right.$, for $k \in \mathbb{N}$, then if $u$ belongs to $W^{k, p}(\Omega)$, the restriction of $H(u)$ to $\Omega$ belongs to $W^{k, p}(\Omega)$ and there exists a constant $C_{k, p}$ such that

$$
\|H(u)\|_{W^{k, p}(\Omega)} \leq C_{k, p}\|u\|_{W^{k, p}(\Omega)} .
$$

Proof. See [8].

\section{Proof of the $H^{2}$ regularity}

In [7], Carbou and Fabrie prove the existence of local strong solutions in $L^{\infty}\left(0, T ; H^{2}\right) \cap L^{2}\left(0, T ; H^{3}\right)$ for the Landau-Lifschitz equation without electric current. Our proof for Theorem 1.1 is basically the same. For the convenience of the reader, we give the complete proof in the present paper, emphasizing the changes due to the electric current.

\subsection{Equivalent system.}

A dissipative term of the form $\|m \times \Delta m\|_{L^{2}}$ appears if we take the inner product in $L^{2}$ of (2) with $\Delta m$. This dissipation is not sufficient to obtain energy estimate in the space $H^{2}(\Omega)$.

We observe that, for $m$ regular enough and $|m|=1$ in $\Omega$, then

$$
m \times(m \times \Delta m)=(m \cdot \Delta m) m-|m|^{2} \Delta m=-\Delta m-|\nabla m|^{2} m .
$$

So, if $m$ is regular enough and satisfies the saturation constraint, then $m$ is solution for the LandauLifschitz equation (2) if and only if $m$ satisfies the following system:

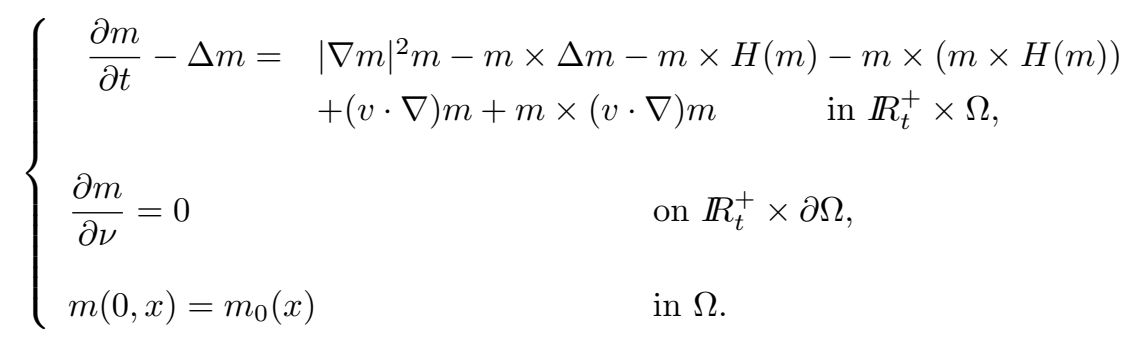

This equation has the advantage to highlight the dissipative term and is more convenient to build regular solutions for (2).

\subsection{Galerkin Approximation for the modified Landau-Lifschitz equation}

We recall that we denote by $V_{n}$ the finite space built on the $n$ first eigenfunctions of the operator $A=$ $-\Delta+I$ with $D(A)=\left\{u \in H^{2}(\Omega)\right.$ such that $\frac{\partial u}{\partial \nu}=0$ on $\left.\partial \Omega\right\}$, and by $P_{n}$ the orthogonal projection from $L^{2}(\Omega)$ on $V_{n}$. We aim to find a solution $m_{n}$ taking its values in $V_{n}$ for the following Galerkin approximation of (4):

$$
\left\{\begin{aligned}
\frac{\partial m_{n}}{\partial t}-\Delta m_{n}= & P_{n}\left(\left|\nabla m_{n}\right|^{2} m_{n}-m_{n} \times \Delta m_{n}\right) \\
& -P_{n}\left(m_{n} \times H\left(m_{n}\right)+m_{n} \times\left(m_{n} \times H\left(m_{n}\right)\right)\right) \\
& +P_{n}\left((v \cdot \nabla) m_{n}+m_{n} \times(v \cdot \nabla) m_{n}\right), \\
m_{n}(0)=P_{n}\left(m_{0}\right) . &
\end{aligned}\right.
$$


Cauchy-Lipschitz theorem ensures the existence of a unique solution of (9) defined on $\left[0, T_{n}[\right.$.

\subsection{1 $\quad L^{2}$ estimate for $(8)$}

Taking the inner product in $L^{2}(\Omega)$ of $(9)$ by $m_{n}$, we obtain

$$
\begin{aligned}
\frac{1}{2} \frac{d}{d t}\left\|m_{n}\right\|_{L^{2}}^{2}+\left\|\nabla m_{n}\right\|_{L^{2}}^{2} & =\int_{\Omega}\left(\left|\nabla m_{n}\right|^{2}\left|m_{n}\right|^{2}+(v \cdot \nabla) m_{n} \cdot m_{n}\right) d x \\
& \leq\left\|\nabla m_{n}\right\|_{L^{2}}^{2}\left\|m_{n}\right\|_{L^{\infty}}^{2}+\|v\|_{L^{\infty}}\left\|\nabla m_{n}\right\|_{L^{2}}\left\|m_{n}\right\|_{L^{2}} \\
& \leq C\left\|m_{n}\right\|_{2, \Omega}^{4}+C\|v\|_{L^{\infty}}\left\|m_{n}\right\|_{2, \Omega}^{2} .
\end{aligned}
$$

\subsection{2 $\quad H^{2}$ estimate for $(8)$}

Now, we take the inner product in $L^{2}(\Omega)$ of $(9)$ with $\Delta^{2} m_{n}$ and we integrate by parts to get

$$
\frac{1}{2} \frac{d}{d t}\left\|\Delta m_{n}\right\|_{L^{2}}^{2}+\left\|\nabla \Delta m_{n}\right\|_{L^{2}}^{2} \leq \sum_{i=1}^{4} I_{i},
$$

where

$$
\begin{gathered}
I_{1}=-\int_{\Omega} \nabla\left(\left|\nabla m_{n}(t)\right|^{2} m_{n}(t)\right) \nabla \Delta m_{n}(t) d x, \\
I_{2}=\int_{\Omega} \nabla\left(m_{n}(t) \times \Delta m_{n}(t)\right) \nabla \Delta m_{n}(t) d x \\
I_{3}=\int_{\Omega} \nabla\left(m_{n}(t) \times H\left(m_{n}(t)\right)+m_{n}(t) \times\left(m_{n}(t) \times H\left(m_{n}(t)\right)\right)\right) \nabla \Delta m_{n}(t) d x, \\
I_{4}=-\int_{\Omega} \nabla\left((v \cdot \nabla) m_{n}(t)+m_{n}(t) \times(v \cdot \nabla) m_{n}(t)\right) \nabla \Delta m_{n}(t) d x,
\end{gathered}
$$

We bound separately each term.

The terms $I_{1}, I_{2}$ and $I_{3}$ are estimated in [7]. For the convenience of the reader, we rewrite these estimates. Using Lemma 2.2, we have

- Estimate on $I_{1}$ :

$$
\begin{aligned}
\left|I_{1}\right| & \leq C \int_{\Omega}\left(\left|m_{n}\left\|\nabla m_{n}\right\| D^{2} m_{n}\right|+\left|\nabla m_{n}\right|^{3}\right)\left|\nabla \Delta m_{n}\right| \\
& \leq C\left(\left\|m_{n}\right\|_{L^{\infty}}\left\|D^{2} m_{n}\right\|_{L^{3}}\left\|\nabla m_{n}\right\|_{L^{6}}+\left\|\nabla m_{n}\right\|_{L^{6}}^{3}\right)\left\|\nabla \Delta m_{n}\right\|_{L^{2}} \\
& \leq C\left\|m_{n}\right\|_{2, \Omega}^{\frac{5}{2}}\left\|\nabla \Delta m_{n}\right\|_{L^{2}}^{\frac{3}{2}}+C\left\|m_{n}\right\|_{2, \Omega}^{3}\left\|\nabla \Delta m_{n}\right\|_{L^{2}} .
\end{aligned}
$$

- Estimate on $I_{2}$ : we remark that $\left(m_{n}(t) \times \nabla \Delta m_{n}(t)\right) \nabla \Delta m_{n}(t)=0$, so

$$
\begin{aligned}
\left|I_{2}\right| & \leq\left\|\Delta m_{n}\right\|_{L^{3}}\left\|\nabla m_{n}\right\|_{L^{6}}\left\|\nabla \Delta m_{n}\right\|_{L^{2}} \\
& \leq C\left\|m_{n}\right\|_{2, \Omega}^{2}\left\|\nabla \Delta m_{n}\right\|_{L^{2}}+C\left\|m_{n}\right\|_{2, \Omega}^{\frac{3}{2}}\left\|\nabla \Delta m_{n}\right\|_{L^{2}}^{\frac{3}{2}} .
\end{aligned}
$$


- Estimate on $I_{3}$

$$
\begin{aligned}
I_{3} & =\int_{\Omega}\left(\nabla m_{n}(t) \times H\left(m_{n}(t)\right) \nabla \Delta m_{n}(t) d x+\int_{\Omega}\left(m_{n}(t) \times \nabla H\left(m_{n}(t)\right) \nabla \Delta m_{n}(t) d x .\right.\right. \\
\left|I_{3}\right| & \leq C \int_{\Omega}\left(1+\left|m_{n}\right|\right)\left(\left|H\left(m_{n}\right)\right|\left|\nabla m_{n}\right|+\left|\nabla H\left(m_{n}\right) \| m_{n}\right|\right)\left|\nabla \Delta m_{n}\right| \\
& \leq C\left(1+\left\|m_{n}\right\|_{L^{\infty}}\right)\left(\left\|H\left(m_{n}\right)\right\|_{L^{3}}\left\|\nabla m_{n}\right\|_{L^{6}}+\left\|\nabla H\left(m_{n}\right)\right\|_{L^{6}}\left\|m_{n}\right\|_{L^{3}}\right)\left\|\nabla \Delta m_{n}\right\|_{L^{2}} \\
& \leq C\left\|\nabla \Delta m_{n}\right\|_{L^{2}}\left(1+\left\|m_{n}\right\|_{2, \Omega}^{3}\right) .
\end{aligned}
$$

- Estimate on $I_{4}$

$$
\begin{aligned}
\left|I_{4}\right| \leq & C \int_{\Omega}\left(\left(1+\left|m_{n}\right|\right)\left(\left|\nabla m_{n}\left\|\nabla v|+| D^{2} m_{n}\right\| v\right|\right)+\left|\nabla m_{n}\right|^{2}|v|\right)\left|\nabla \Delta m_{n}\right| \\
\leq & C\left(1+\left\|m_{n}\right\|_{L^{\infty}}\right)\left(\left\|\nabla m_{n}\right\|_{L^{6}}\|\nabla v\|_{L^{3}}+\left\|D^{2} m_{n}\right\|_{L^{2}}\|v\|_{L^{\infty}}\right)\left\|\nabla \Delta m_{n}\right\|_{L^{2}} \\
& +C\left\|\nabla m_{n}\right\|_{L^{4}}^{2}\|v\|_{L^{\infty}}\left\|\nabla \Delta m_{n}\right\|_{L^{2}} \\
\leq & C\left(\|\nabla v\|_{L^{3}}+\|v\|_{L^{\infty}}\right)\left(1+\left\|m_{n}\right\|_{2, \Omega}^{2}\right)\left\|\nabla \Delta m_{n}\right\|_{L^{2}}
\end{aligned}
$$

By addition of all these estimates and using Young inequality, we obtain the following inequality

$$
\frac{d}{d t}\left\|\Delta m_{n}\right\|_{L^{2}}^{2}+\left\|\nabla \Delta m_{n}\right\|_{L^{2}}^{2} \leq C\left(1+\|\nabla v\|_{L^{3}}^{2}+\|v\|_{L^{\infty}}^{2}\right)\left(1+\left\|m_{n}\right\|_{2, \Omega}^{10}\right) .
$$

\subsubsection{Uniform estimate on the Galerkin approximation}

Summing Inequalities (10) and (11), we obtain that there exists a constant $C$ such that

$$
\frac{d}{d t}\left\|m_{n}\right\|_{2, \Omega}^{2}+\left\|\nabla m_{n}\right\|_{L^{2}}^{2}+\left\|\nabla \Delta m_{n}\right\|_{L^{2}}^{2} \leq C\left(1+\|\nabla v\|_{L^{3}}^{2}+\|v\|_{L^{\infty}}^{2}\right)\left(1+\left\|m_{n}\right\|_{2, \Omega}^{10}\right),
$$

where $C$ does not depend on $n$. We denote:

$$
c(t)=C\left(1+\|\nabla v(t)\|_{L^{3}}^{2}+\|v(t)\|_{L^{\infty}}^{2}\right) .
$$

From the assumptions about $v, c \in \mathcal{C}^{0}\left(\mathbb{R}^{+}\right)$.

In addition, $m_{n}(0)=P_{n}\left(m_{0}\right)$, and since $m_{0}$ satisfies the compatibility condition (3), by Proposition 1 , we obtain that for all $n$ :

$$
\left\|m_{n}(0)\right\|_{2, \Omega} \leq C\left\|m_{0}\right\|_{2, \Omega} .
$$

We set $y_{n}(t)=\left\|m_{n}\right\|_{2, \Omega}^{2}$. We have proven that for all $n$,

$$
\left\{\begin{array}{l}
\frac{d}{d t} y_{n}(t) \leq c(t)\left(1+y_{n}^{5}(t)\right), \\
y_{n}(0) \leq C\left\|m_{0}\right\|_{2, \Omega}^{2}
\end{array}\right.
$$

We consider $z$ the maximal solution of the o.d.e.:

$$
\left\{\begin{array}{l}
\frac{d}{d t} z(t)=c(t)\left(1+z^{5}(t)\right), \\
z(0)=C\left\|m_{0}\right\|_{2, \Omega}^{2}
\end{array}\right.
$$


and we denote by $T^{*}$ the maximal existence time of $z$. By the comparison lemma, we have:

$$
\forall t<T^{*}, \quad \forall n, y_{n}(t) \leq z(t) .
$$

Therefore, for all $T<T^{*}$, there exists a constant $C(T)$ such that

$$
\sup _{t \leq T}\left\|m_{n}(t)\right\|_{H^{2}(\Omega)}^{2} \leq C(T),
$$

and by integration of (12) on the interval $[0, T]$ we obtain

$$
\int_{0}^{T}\left(\left\|\nabla m_{n}(t)\right\|_{L^{2}}^{2}+\left\|\nabla \Delta m_{n}(t)\right\|_{L^{2}}^{2}\right) d t \leq C(T) .
$$

By equation (9) we conclude that

$$
\sup _{t \leq T}\left\|\frac{\partial}{\partial t} m_{n}(t)\right\|_{L^{2}}^{2} \leq C(T),
$$

and

$$
\int_{0}^{T}\left\|\frac{\partial}{\partial t} \nabla m_{n}(\tau)\right\|_{L^{2}}^{2} d \tau \leq C(T) .
$$

\subsection{Limit when $n$ tends to $+\infty$}

These uniform estimates insure the existence of a subsequence $\left(m_{n_{k}}\right)$ and a function $m$ such that

- $m_{n_{k}} \rightarrow m$ in $L^{2}\left(0, T ; H^{3}(\Omega)\right)$ weak,

- $m_{n_{k}} \rightarrow m$ in $L^{\infty}\left(0, T ; H^{2}(\Omega)\right)$ weak $*$,

- $\frac{\partial m_{n_{k}}}{\partial t} \rightarrow \frac{\partial m}{\partial t}$ in $L^{2}\left(0, T ; H^{1}(\Omega)\right)$ weak.

Thanks to Aubin-Simon compactness lemma (see [2] and [12]), we can conclude that

$$
m_{n_{k}} \longrightarrow m \text { in } L^{\infty}\left(0, T ; H^{2}(\Omega)\right) \text { strong, }
$$

and

$$
H\left(m_{n_{k}}\right) \longrightarrow H(m) \text { in } L^{\infty}\left(0, T ; H^{2}(\Omega)\right) \text { strong }
$$

because $H$ is a continuous map on Sobolev spaces $H^{s}(\Omega)$ for $s=0,1,2$.

Taking the limit in (9), we obtain that $m$ satisfies

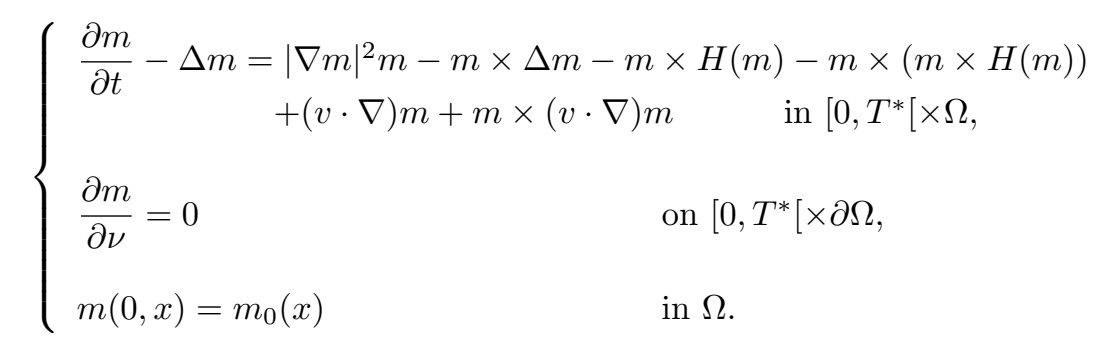

In addition, we remark that from Theorem II.5.14 in [4], then

$$
m \in \mathcal{C}^{0}\left(\left[0, T^{*}\left[; H^{2}(\Omega)\right) .\right.\right.
$$




\subsection{Conservation of the ponctual norm}

We prove now that the solution $m$ constructed in the previous part satisfies the physical constraint $|m|=1$, so that $m$ satisfies Landau-Lifschitz equation. Contrary to [7], a transport term due to electric current appears in equation (4).

Using the scalar product in $\mathbb{R}^{3}$ of (4) by $m$, we get

$$
\frac{1}{2} \frac{d}{d t}|m|^{2}-m \cdot \Delta m-|\nabla m|^{2}|m|^{2}-m .(v \cdot \nabla) m=0 \quad \text { in }[0, T[\times \Omega .
$$

For $d \leq 3$ and for all $u \in L^{\infty}\left(0, T ; H^{2}(\Omega)\right)$ we have

$$
\Delta|u|^{2}=2 u \cdot \Delta u+2|\nabla u|^{2}
$$

and $m \cdot(v \cdot \nabla) m=\frac{1}{2}(v \cdot \nabla)|m|^{2}$, then $|m|^{2}$ satisfies the following equation

$$
\frac{d}{d t}|m|^{2}-\Delta|m|^{2}-2|\nabla m|^{2}\left(|m|^{2}-1\right)-\frac{1}{2} v \cdot \nabla|m|^{2}=0 .
$$

Taking $a=|m|^{2}-1$, then $a$ satisfies the system below

$$
\begin{cases}\frac{\partial a}{\partial t}-\Delta a-2|\nabla m|^{2} a-\frac{1}{2}(v \cdot \nabla) a=0 & \text { in } \Omega \\ \frac{\partial a}{\partial \nu}=0 & \text { on } \partial \Omega, \\ a(0)=\left|m_{0}\right|^{2}-1=0 & \text { in } \Omega .\end{cases}
$$

Taking the inner product of this equation by $a$, we obtain that

$$
\begin{aligned}
\frac{1}{2} \frac{d}{d t}\|a\|_{L^{2}}^{2}+\|\nabla a\|_{L^{2}}^{2} & =2 \int_{\Omega}|\nabla m|^{2} a^{2}+\frac{1}{2} \int_{\Omega}(v \cdot \nabla) a \cdot a \\
& \leq C\|\nabla m\|_{L^{\infty}}^{2}\|a\|_{L^{2}}^{2}+\frac{1}{2}\|v\|_{L^{\infty}}\|a\|_{L^{2}}\|\nabla a\|_{L^{2}},
\end{aligned}
$$

and by absorbing $\|\nabla a\|_{L^{2}}$, we obtain that

$$
\frac{d}{d t}\|a\|_{L^{2}}^{2}+\|\nabla a\|_{L^{2}}^{2} \leq C\left(\|\nabla m\|_{L^{\infty}}^{2}+\|v\|_{L^{\infty}}^{2}\right)\|a\|_{L^{2}}^{2} .
$$

Since $m \in L^{2}\left(0, T ; H^{3}(\Omega)\right)$, the map $t \mapsto\left(\|\nabla m\|_{L^{\infty}}^{2}+\|v\|_{L^{\infty}}^{2}\right)$ is in $L^{1}([0, T])$. So by using the Gronwall lemma, since $a(0, x)=0$, we can conclude that $\|a\|_{L^{2}}^{2}=0$ and therefore the ponctual norm of $m$ is conserved.

Under the assumption $|m|=1$, the equations (2) and (4) are equivalent. Hence, we have proven the existence of a strong solution for $(2)$ in the space $L^{\infty}\left(0, T ; H^{2}(\Omega)\right) \cap L^{2}\left(0, T ; H^{3}(\Omega)\right)$ for $T<T^{*}$. It remains to prove that this solution is unique.

\subsection{Uniqueness for the strong solution of (2)}

Let $m^{1}$ and $m^{2}$ in $L^{\infty}\left(0, T ; H^{2}\left(\Omega ; S^{2}\right)\right) \cap L^{2}\left(0, T ; H^{3}(\Omega)\right)$ satisfying (2). Since (2) preserves the saturation constraint (1) for the strong solutions, they satisfy (4). We denote $w=m^{1}-m^{2}$. Then $w \in L^{\infty}\left(0, T ; H^{2}\left(\Omega ; S^{2}\right)\right) \cap L^{2}\left(0, T ; H^{3}(\Omega)\right)$ and is solution to

$$
\left\{\begin{array}{l}
\frac{\partial w}{\partial t}-\Delta w=T_{1}+T_{2}+T_{3}+T_{4} \\
\frac{\partial w}{\partial \nu}=0 \text { on } \partial \Omega \\
w(0, x)=0
\end{array}\right.
$$


where

- $T_{1}=-m^{2} \times \Delta w$

- $T_{2}=m^{2}\left(\nabla w \cdot \nabla\left(m^{1}+m^{2}\right)\right)+(v \cdot \nabla) w-m^{2} \times(v \cdot \nabla) w$,

- $T_{3}=\left|\nabla m^{1}\right|^{2} w-w \times \Delta m^{1}+w \times(v \cdot \nabla) m^{1}$,

- $T_{4}=-m^{1} \times H(w)-w \times H\left(m^{1}\right)-w \times\left(m^{1} \times H\left(m^{1}\right)\right)-m^{2} \times\left(w \times H\left(m^{1}\right)\right)-m^{2} \times\left(m^{2} \times H(w)\right)$.

We take the inner product of (16) with $w$. Since

$$
\int_{\Omega} T_{1} w=-\int_{\Omega} \nabla m^{2} \times v \cdot \nabla w
$$

and using that $\left\|m^{1}\right\|_{L^{\infty}}=\left\|m^{2}\right\|_{L^{\infty}}=1$, we obtain that

$$
\begin{aligned}
\frac{1}{2} \frac{d}{d t}\|w\|_{L^{2}}^{2}+\|\nabla w\|_{L^{2}}^{2} \leq & \|w\|_{L^{2}}^{2}\left(\left\|\nabla m^{1}\right\|_{L^{\infty}}+1+\left\|H\left(m^{1}\right)\right\|_{L^{\infty}}\right) \\
& +c\|w\|_{L^{2}}\|\nabla w\|_{L^{2}}\left[1+\left\|\nabla m^{1}\right\|_{L^{\infty}}+\left\|\nabla m^{2}\right\|_{L^{\infty}}\right. \\
& \left.+\|v\|_{L^{\infty}}\right] .
\end{aligned}
$$

So by using the Young inequality, we obtain that

$$
\frac{d}{d t}\|w\|_{L^{2}}^{2}+\|\nabla w\|_{L^{2}}^{2} \leq C\left(1+\left\|m^{1}\right\|_{H^{3}(\Omega)}^{2}+\left\|m^{2}\right\|_{H^{3}(\Omega)}^{2}+\|v\|_{L^{\infty}}^{2}\right)\|w\|_{L^{2}}^{2},
$$

and since $m^{1}$ and $m^{2}$ are in $L^{2}\left(0, T ; H^{3}(\Omega)\right)$, we conclude by the Gronwall lemma that for all $t \geq 0$,

$$
\|w(t)\|_{L^{2}}^{2} \leq\|w(0)\|_{L^{2}}^{2} \exp \left(C \int_{0}^{t}\left(1+\left\|m^{1}(\tau)\right\|_{H^{3}(\Omega)}^{2}+\left\|m^{2}(\tau)\right\|_{H^{3}(\Omega)}^{2}+\|v(\tau)\|_{L^{\infty}}^{2}\right) d \tau\right),
$$

and since $w(0)=0$, we obtain that $w=0$ for all $t$, which concludes the proof of Theorem 1.1.

\section{Study of the case $s=3$}

In order to obtain more regular solutions, it would be standard to multiply the Galerkin approximation (9) by $\Delta^{3} m_{n}$ to obtain a $H^{3}$-estimate. Unfortunately, the non local term $H\left(m_{n}\right)$ does not satisfy the homogeneous Neumann boundary condition, so that the necessary double integration by parts is not possible. Therefore, the $H^{3}$ regularity is obtained by derivation of (9) with respect to $t$ in order to obtain a $H^{1}$ estimate on $\partial_{t} m$. We conclude the proof by a bootstrap argument to obtain that $\Delta m$ and $\partial_{t} m$ have the same regularity.

\section{1 $H^{1}$ regularity for $\partial_{t} m$}

We differentiate the Galerkin approximation (9) with respect to $t$. Denoting by $w_{1, n}=\partial_{t} m_{n}$ the time derivative of $m_{n}$, we obtain that

$$
\frac{\partial w_{1, n}}{\partial t}-\Delta w_{1, n}=\sum_{i=1}^{6} T_{i}
$$

with

- $T_{1}=-P_{n}\left(m_{n} \times \Delta w_{1, n}\right)$

- $T_{2}=P_{n}\left(\left|\nabla m_{n}\right|^{2} w_{1, n}+2\left(\nabla m_{n} \cdot \nabla w_{1, n}\right) m_{n}-w_{1, n} \times \Delta m_{n}\right)$ 
- $T_{3}=-P_{n}\left(w_{1, n} \times H\left(m_{n}\right)+m_{n} \times H\left(w_{1, n}\right)\right)$,

- $T_{4}=-P_{n}\left(w_{1, n} \times\left(m_{n} \times H\left(m_{n}\right)\right)+m_{n} \times\left(w_{1, n} \times H\left(m_{n}\right)\right)+m_{n} \times\left(m_{n} \times H\left(w_{1, n}\right)\right)\right)$

- $T_{5}=P_{n}\left((v \cdot \nabla) w_{1, n}+w_{1, n} \times(v \cdot \nabla) m_{n}+m_{n} \times(v \cdot \nabla) w_{1, n}\right)$

- $T_{6}=P_{n}\left(\left(\partial_{t} v \cdot \nabla\right) m_{n}+m_{n} \times\left(\partial_{t} v \cdot \nabla\right) m_{n}\right)$

We multiply (18) by $-\Delta w_{1, n}$ and we integrate on $\Omega$. Since $m_{n} \times \Delta w_{1, n} \cdot \Delta w_{1, n}=0$, we obtain that:

$$
\frac{1}{2} \frac{d}{d t}\left\|\nabla w_{1, n}\right\|_{L^{2}}^{2}+\left\|\Delta w_{1, n}\right\|_{L^{2}}^{2} \leq\left\|\Delta w_{1, n}\right\|_{L^{2}} \sum_{i=2}^{6}\left\|T_{i}\right\|_{L^{2}} .
$$

Using the continuity of the operator $H$ on $H^{k}(\Omega)$ for $k=0,1,2$, we estimate each term:

$$
\begin{aligned}
\left\|T_{2}\right\|_{L^{2}} \leq & \left\|\nabla m_{n}\right\|_{L^{6}}^{2}\left\|w_{1, n}\right\|_{L^{6}}+\left\|\nabla m_{n}\right\|_{L^{\infty}(\Omega)}\left\|\nabla w_{1, n}\right\|_{L^{2}(\Omega)}\left\|m_{n}\right\|_{L^{\infty}(\Omega)} \\
& +\left\|w_{1, n}\right\|_{L^{6}(\Omega)}\left\|\Delta m_{n}\right\|_{L^{3}(\Omega)} \\
\leq & C\left(\left\|\nabla w_{1, n}\right\|_{L^{2}}+\left\|w_{1, n}\right\|_{L^{2}}\right)\left(1+\left\|m_{n}\right\|_{H^{2}}^{2}+\left\|m_{n}\right\|_{H^{3}(\Omega)}\right) \\
\left\|T_{3}+T_{4}\right\|_{L^{2}} \leq & C\left\|w_{1, n}\right\|_{L^{2}(\Omega)}\left(1+\left\|m_{n}\right\|_{H^{2}(\Omega)}^{2}\right) \\
\left\|T_{5}\right\|_{L^{2}} \leq & \left\|\nabla w_{1, n}\right\|_{L^{2}}\left(\left\|m_{n}\right\|_{L^{\infty}(\Omega)}+1\right)\|v\|_{L^{\infty}(\Omega)}+\left\|w_{1, n}\right\|_{L^{6}(\Omega)}\|v\|_{L^{\infty}(\Omega)}\left\|\nabla m_{n}\right\|_{L^{3}(\Omega)} \\
\leq & C\left(\left\|w_{1, n}\right\|_{L^{2}}+\left\|\nabla w_{1, n}\right\|_{L^{2}}\right)\left(\left\|m_{n}\right\|_{H^{2}(\Omega)}+1\right)\|v\|_{L^{\infty}(\Omega)} \\
\left\|T_{6}\right\|_{L^{2}} \leq & \left\|\partial_{t} v\right\|_{L^{2}(\Omega)}\left\|\nabla m_{n}\right\|_{L^{\infty}(\Omega)}\left(1+\left\|m_{n}\right\|_{L^{\infty}(\Omega)}^{2}\right) \\
\leq & C\left\|\partial_{t} v\right\|_{L^{2}(\Omega)}\left(1+\left\|m_{n}\right\|_{H^{2}(\Omega)}^{2}\right)\left\|m_{n}\right\|_{H^{3}(\Omega)} .
\end{aligned}
$$

Since $v$ is sufficiently regular, by absorbing $\left\|\Delta w_{1, n}\right\|_{L^{2}}$, we obtain that:

$$
\frac{d}{d t}\left\|\nabla w_{1, n}\right\|_{L^{2}}^{2}+\left\|\Delta w_{1, n}\right\|_{L^{2}}^{2} \leq g_{1}^{n}(t)\left\|\nabla w_{1, n}\right\|_{L^{2}}^{2}+g_{2}^{n}(t),
$$

where

$$
g_{1}^{n}(t)=C\left(1+\left\|m_{n}\right\|_{H^{2}(\Omega)}^{2}\right)\left\|m_{n}\right\|_{H^{3}(\Omega)}^{2}+C\left(1+\left\|m_{n}\right\|_{H^{2}(\Omega)}^{4}\right),
$$

and

$$
g_{2}^{n}(t)=C\left\|w_{1, n}\right\|_{L^{2}}^{2}\left(1+\left\|m_{n}\right\|_{H^{3}(\Omega)}^{2}+\left\|m_{n}\right\|_{H^{2}(\Omega)}^{4}\right)+C\left\|\partial_{t} v\right\|_{L^{2}}^{2}\left(1+\left\|m_{n}\right\|_{H^{2}(\Omega)}^{4}\right)\left\|m_{n}\right\|_{H^{3}(\Omega)} .
$$

Let us now estimate the initial value of $\partial_{t} m_{n}$. By Equation (9) taken at $t=0$, we have:

$$
\begin{aligned}
w_{1, n}(0)= & \partial_{t} m_{n}(0) \\
= & P_{n}\left(\Delta m_{n}(0)+\left|\nabla m_{n}(0)\right|^{2} m_{n}(0)-m_{n}(0) \times \Delta m_{n}(0)\right) \\
& -P_{n}\left(m_{n}(0) \times H\left(m_{n}(0)\right)+m_{n}(0) \times\left(m_{n}(0) \times H\left(m_{n}(0)\right)\right)\right) \\
& +P_{n}\left(\left(v_{0} \cdot \nabla\right) m_{n}(0)+m_{n}(0) \times\left(v_{0} \cdot \nabla\right) m_{n}(0) .\right)
\end{aligned}
$$


(we recall that $\left.v_{0}(x)=v(0, x)\right)$.

Using Proposition 1 we can estimate the $H^{1}$ norm of $w_{1, n}(0)$ without compatibility condition on the following way:

$$
\begin{aligned}
\left\|w_{1, n}(0)\right\|_{H^{1}(\Omega)} \leq & \left\|\Delta m_{n}(0)+\left|\nabla m_{n}(0)\right|^{2} m_{n}(0)-m_{n}(0) \times \Delta m_{n}(0)\right\|_{H^{1}(\Omega)} \\
& +\left\|m_{n}(0) \times H\left(m_{n}(0)\right)+m_{n}(0) \times\left(m_{n}(0) \times H\left(m_{n}(0)\right)\right)\right\|_{H^{1}(\Omega)} \\
& +\left\|\left(v_{0} \cdot \nabla\right) m_{n}(0)+m_{n}(0) \times\left(v_{0} \cdot \nabla\right) m_{n}(0)\right\|_{H^{1}(\Omega)} \\
\leq & C\left(1+\left\|m_{n}(0)\right\|_{H^{3}(\Omega)}^{3}\right) \\
\leq & C\left(1+\left\|m_{0}\right\|_{H^{3}(\Omega)}^{3}\right)
\end{aligned}
$$

using the compatibility condition (3) and Proposition 1.

Let us consider $z_{n}(t)=\left\|\nabla w_{1, n}\right\|_{H^{1}(\Omega)}^{2}$ then we have

$$
\left\{\begin{array}{l}
z_{n}^{\prime}(t) \leq g_{1}^{n}(t) z_{n}(t)+g_{2}^{n}(t), \\
z_{n}(0) \leq C\left(1+\left\|m_{0}\right\|_{H^{3}(\Omega)}^{3}\right) .
\end{array}\right.
$$

Let $\xi_{n}$ be the solution of

We have:

$$
\left\{\begin{array}{l}
\xi_{n}^{\prime}(t)=g_{1}^{n}(t) \xi_{n}(t)+g_{2}^{n}(t), \\
\xi_{n}(0)=C\left(1+\left\|m_{0}\right\|_{H^{3}(\Omega)}^{3}\right) .
\end{array}\right.
$$

$$
\xi_{n}(t)=\xi_{n}(0) \exp \left(\int_{0}^{t} g_{1}^{n}(\tau) d \tau\right)+\int_{0}^{t} g_{2}^{n}(\tau) \exp \left(\int_{\tau}^{t} g_{1}^{n}(s) d s\right) d \tau .
$$

From the estimates (13) and (14), we obtain that for all $T<T^{*}$, there exists a constant $C(T)$ independent of $n$ such that:

$$
\int_{0}^{T} g_{1}^{n}(\tau) d \tau \leq C(T) \text { and } \int_{0}^{T} g_{2}^{n}(\tau) d \tau \leq C(T),
$$

so that we deduce that

$$
\forall T<T^{*}, \exists C(T), \forall n, \quad \xi_{n}(T) \leq C(T) .
$$

Therefore, by the Comparison Lemma 2.4, we obtain that for every $T<T^{*}$, there exists a constant $C(T)$ independent of $n$ such that

$$
\sup _{t \leq T}\left\|w_{1, n}(t)\right\|_{H^{1}(\Omega)}^{2} \leq C
$$

and by integration of (19) on the interval $[0, T]$ we obtain

$$
\int_{0}^{T}\left\|\Delta w_{1, n}(t)\right\|_{L^{2}}^{2} d t \leq C
$$

From these inequalities, we can conclude that there exist a subsequence $\left(w_{1, n_{k}}\right)$ such that

- $w_{1, n_{k}} \rightarrow \partial_{t} m$ in $L^{2}\left(0, T ; H^{2}(\Omega)\right)$ weak,

- $w_{1, n_{k}} \rightarrow \partial_{t} m$ in $L^{\infty}\left(0, T ; H^{1}(\Omega)\right)$ weak*.

Therefore we obtain that $\partial_{t} m$ is bounded in the space $L^{\infty}\left(0, T ; H^{1}(\Omega)\right) \cap L^{2}\left(0, T ; H^{2}(\Omega)\right)$ for all $T<T^{*}$. Let us show that this estimate yields that $m \in L^{\infty}\left(0, T ; H^{3}(\Omega)\right) \cap L^{2}\left(0, T ; H^{4}(\Omega)\right)$. 


\subsection{Regularity of $\Delta m$}

In this paragraph, we will prove that $\Delta m \in L^{\infty}\left(0, T ; H^{1}(\Omega)\right) \cap L^{2}\left(0, T ; H^{2}(\Omega)\right)$, so that $m \in$ $L^{\infty}\left(0, T ; H^{3}(\Omega)\right) \cap L^{2}\left(0, T ; H^{4}(\Omega)\right)$. By the equation, we have:

$$
\Delta m-m \times \Delta m=\partial_{t} m-|\nabla m|^{2} m+m \times(H(m)+m \times H(m))-(v \cdot \nabla) m-m \times(v \cdot \nabla) m .
$$

Let us consider $g_{m}$ the map defined by $\xi \longrightarrow \xi-m \times \xi$. This map is linear bijective which inverse $\psi_{m}=g_{m}^{-1}$ is given by

$$
\psi_{m}(y)=\xi=\frac{1}{2}(y+m \times y+(m . y) m) .
$$

Since $|m|=1$ then $\psi_{m}\left(\partial_{t} m\right)=\frac{1}{2}\left(\partial_{t} m+m \times \partial_{t} m\right), \psi_{m}(m)=m$ and we obtain that:

$$
\Delta m=\frac{1}{2}\left(\partial_{t} m+m \times \partial_{t} m\right)-|\nabla m|^{2} m+W,
$$

where $W=m \times(m \times H(m))-m \times(v \cdot \nabla) m$.

Lemma 4.1. For all $T<T^{*}, \psi_{m}\left(\partial_{t} m\right) \in L^{\infty}\left(0, T ; H^{1}(\Omega)\right) \cap L^{2}\left(0, T ; H^{2}(\Omega)\right)$.

Proof. We recall that

$$
\psi_{m}\left(\partial_{t} m\right)=\frac{1}{2}\left(\partial_{t} m+m \times \partial_{t} m\right) .
$$

In the previous section, we have proven that $\partial_{t} m \in L^{\infty}\left(0, T ; H^{1}(\Omega)\right)$ and $m \in L^{\infty}\left(0, T ; H^{2}(\Omega)\right)$. So by Lemma 2.3 ,

$$
\psi_{m}\left(\partial_{t} m\right) \in L^{\infty}\left(0, T ; H^{1}(\Omega)\right) .
$$

Now, $H^{2}(\Omega)$ is an algebra, so since $m \in L^{\infty}\left(0, T ; H^{2}(\Omega)\right)$ and $\partial_{t} m \in L^{2}\left(0, T ; H^{2}(\Omega)\right)$, we obtain that

$$
\psi_{m}\left(\partial_{t} m\right) \in L^{2}\left(0, T ; H^{2}(\Omega)\right) .
$$

Lemma 4.2. For all $T<T^{*}, W \in L^{\infty}\left(0, T ; H^{1}(\Omega)\right) \cap L^{2}\left(0, T ; H^{2}(\Omega)\right)$.

Proof. We know that $m \in L^{\infty}\left(0, T ; H^{2}(\Omega)\right)$. From Proposition 1 , the same holds for $H(m)$, so since $L^{\infty}\left(0, T ; H^{2}(\Omega)\right)$ is an algebra,

$$
m \times(m \times H(m)) \in L^{\infty}\left(0, T ; H^{2}(\Omega)\right) .
$$

So a fortiori this term belongs to $L^{\infty}\left(0, T ; H^{1}(\Omega)\right) \cap L^{2}\left(0, T ; H^{2}(\Omega)\right)$.

Concerning the other term, $m$ and $v$ are bounded in $L^{\infty}\left(0 ; T ; H^{2}(\Omega)\right)$ and $\nabla m$ is bounded in $L^{\infty}\left(0, T ; H^{1}(\Omega)\right)$. So by Lemma 2.3 ,

$$
m \times(v \cdot \nabla) m \in L^{\infty}\left(0, T ; H^{1}(\Omega)\right) .
$$

On the other hand, $m$ and $v$ are bounded in $L^{\infty}\left(0 ; T ; H^{2}(\Omega)\right)$ and $\nabla m$ is bounded in $L^{2}\left(0, T ; H^{2}(\Omega)\right)$, thus, since $H^{2}(\Omega)$ is an algebra,

$$
m \times(v \cdot \nabla) m \in L^{2}\left(0, T ; H^{2}(\Omega)\right) .
$$

We aim to deduce from (21) more regularity for $m$. We have:

$$
\left|\nabla\left(|\nabla m|^{2} m\right)\right| \leq|\nabla m|^{3}+2|m||\nabla m|\left|D^{2} m\right|
$$




$$
\begin{aligned}
\left\|\nabla\left(|\nabla m|^{2} m\right)\right\|_{L^{2}} & \leq C\left(\|\nabla m\|_{L^{6}}^{3}+\|m\|_{L^{\infty}}\|\nabla m\|_{L^{6}}\left\|D^{2} m\right\|_{L^{3}}\right) \\
& \leq C\|m\|_{H^{2}}^{3}+C\|m\|_{H^{2}}^{\frac{5}{2}}\|m\|_{H^{3}}^{\frac{1}{2}}
\end{aligned}
$$

by Proposition 2.1. Since $m \in L^{\infty}\left(0, T ; H^{2}(\Omega)\right) \cap L^{2}\left(0, T ; H^{3}(\Omega)\right)$, then

$$
|\nabla m|^{2} m \in L^{4}\left(0, T ; H^{1}(\Omega)\right) .
$$

Using Lemmas 4.1 and 4.2 , we obtain then from (21) that:

$$
\Delta m \in L^{4}\left(0, T ; H^{1}(\Omega)\right), \text { so } m \in L^{4}\left(0, T ; H^{3}(\Omega)\right) .
$$

Now, $\nabla m \in L^{4}\left(0, T ; H^{2}(\Omega)\right)$ and $m \in L^{\infty}\left(0, T ; H^{2}(\Omega)\right)$. So

$$
|\nabla m|^{2} m \in L^{2}\left(0, T ; H^{2}(\Omega)\right)
$$

By using lemmas 4.1 and 4.2 , we obtain then that

$$
\Delta m \in L^{2}\left(0, T ; H^{2}(\Omega)\right),
$$

and so

$$
m \in L^{2}\left(0, T ; H^{4}(\Omega)\right) .
$$

By Theorem II.5.14 in [4], since we know that $\partial_{t} m \in L^{2}\left(0, T ; H^{2}(\Omega)\right)$, we obtain that

$$
m \in \mathcal{C}^{0}\left(0, T ; H^{3}(\Omega)\right) .
$$

This conclude the proof of Theorem 1.2.

\section{$5 \quad$ Very regular solutions}

It is not possible to obtain $H^{2}$ estimates on $w_{1, n}=\partial_{t} m_{n}$ using Equation (18). Indeed, we would need a uniform estimate on the initial value $w_{1, n}(0)$ in the $H^{2}$ norm, and using Proposition 1, it would be necessary to check a compatibility condition of the form:

$$
\begin{aligned}
& \frac{\partial}{\partial \nu}\left(\Delta m_{n}(0)+\left|\nabla m_{n}(0)\right|^{2} m_{n}(0)-m_{n}(0) \times \Delta m_{n}(0)-m_{n}(0) \times H\left(m_{n}(0)\right)\right. \\
& \left.+m_{n}(0) \times\left(m_{n}(0) \times H\left(m_{n}(0)\right)\right)+\left(v_{0} \cdot \nabla\right) m_{n}(0)+m_{n}(0) \times\left(v_{0} \cdot \nabla\right) m_{n}(0)\right)=0 .
\end{aligned}
$$

Because of the non local term $H\left(m_{n}(0)\right)$, this condition cannot be satisfied for all $n$.

We assume that $m_{0} \in H^{4}\left(\Omega ; S^{2}\right)$ and satisfies the compatibility condition at order one (see Definition 1.3). In order to obtain more regular solutions, our strategy is the following.

1. We compute the equation (23) (see below) satisfied by the time derivative $\partial_{t} m$.

2. We construct a solution $w_{1}$ for this equation in $L^{\infty}\left(0, T ; H^{2}\right) \cap L^{2}\left(0, T ; H^{3}\right)$. At this step we need a compatibility condition at the boundary for $\partial_{t} m(t=0)$.

3. We already know that $\partial_{t} m \in L^{\infty}\left(0, T ; H^{1}\right) \cap L^{2}\left(0, T ; H^{2}\right)$ and satisfies (23). In addition, we show a uniqueness result for the solutions of (23), so that $w_{1}=\partial_{t} m$.

4. Writing $\Delta m$ in function of $\partial_{t} m$, we prove that $\Delta m \in L^{\infty}\left(0, T ; H^{2}\right) \cap L^{2}\left(0, T ; H^{3}\right)$, so that $m \in L^{\infty}\left(0, T ; H^{4}\right) \cap L^{2}\left(0, T ; H^{5}\right)$. 
If $m_{0} \in H^{5}\left(\Omega ; S^{2}\right)$ satisfies the compatibility condition at order one, we obtain the desired regularity by differentiating the Galerkin approximation of $\left(^{*}\right)$ with respect to time (in the same spirit we obtained the $H^{3}$ regularity for $m$ in the previous part).

This strategy will be used at any order to obtain very regular solutions for the Landau-Lifschitz equation.

We will detail this process at any order by proving by induction the following property $\mathcal{P}(k)$ :

If $m_{0} \in H^{2 k}\left(\Omega ; S^{2}\right)$ satisfies the compatibility condition at order $k-1$, if for all $j \leq k-1$, $\partial_{t}^{j} v \in \mathcal{C}^{0}\left(\mathbb{R}^{+} ; H^{2 k-2 j}(\Omega)\right)$, then the solution $m$ of the Landau-Lifschitz equation (2) with initial data $m_{0}$ satisfies:

$\mathcal{P}(k)$

$$
\forall T<T^{*}, \forall i \in\{0, \ldots k\}, \quad \partial_{t}^{i} m \in L^{\infty}\left(0, T ; H^{2 k-2 i}(\Omega)\right) \cap L^{2}\left(0, T ; H^{2 k-2 i+1}(\Omega)\right) .
$$

If in addition $m_{0} \in H^{2 k+1}\left(\Omega ; S^{2}\right)$, if for all $j \in\{0, \ldots, k\}, \partial_{t}^{j} v \in \mathcal{C}^{0}\left(\mathbb{R}^{+} ; H^{2 k-2 j+1}(\Omega)\right)$, then $m$ satisfies:

$$
\forall T<T^{*}, \forall i \in\{0, \ldots k\}, \partial_{t}^{i} m \in L^{\infty}\left(0, T ; H^{2 k-2 i+1}(\Omega)\right) \cap L^{2}\left(0, T ; H^{2 k-2 i+2}(\Omega)\right)
$$

This property is proven for $k=1$ in Sections 3 and 4 .

Let $k \geq 1$. Let us assume that $\mathcal{P}(k)$ is true, and let us establish $\mathcal{P}(k+1)$.

Let $m_{0} \in H^{2(k+1)}\left(\Omega ; S^{2}\right)$ satisfying the compatibility condition at order $k$. By Property $\mathcal{P}(k)$ we already know that

$$
\forall T<T^{*}, \forall i \in\{0, \ldots k\}, \partial_{t}^{i} m \in L^{\infty}\left(0, T ; H^{2 k-2 i+1}(\Omega)\right) \cap L^{2}\left(0, T ; H^{2 k-2 i+2}(\Omega)\right) .
$$

We denote by $w_{j}=\partial_{t}^{j} m$ for $j \in\{0, \ldots, k\}$. By differentiating $(2) j$ times with respect to $t$, we obtain that $w_{j}$ satisfies:

$$
\left\{\begin{array}{l}
\frac{\partial w_{j}}{\partial t}-\Delta w_{j}=-m \times \Delta w_{j}+K_{m}\left(\nabla w_{j}\right)+L_{m}\left(w_{j}\right)+F_{j} \\
\frac{\partial w_{j}}{\partial \nu}=0 \quad \text { on } \mathbb{R}^{+} \times \partial \Omega \\
w_{j}(t=0)=V_{j},
\end{array}\right.
$$

where

$$
\begin{array}{ll}
K_{m}(\nabla w)=\quad & 2(\nabla m \cdot \nabla w) m+(v \cdot \nabla) w+m \times(v \cdot \nabla) w, \\
L_{m}(w)=\quad & |\nabla m|^{2} w-w \times \Delta m-w \times H(m)-m \times H(w)-w \times(m \times H(m)) \\
& -m \times(w \times H(m))-m \times(m \times H(w))+w \times(v \cdot \nabla) m, \\
F_{j}=\quad & \sum_{\alpha \in \bar{A}_{j}} C^{\alpha}\left(\left(\nabla w_{\alpha_{1}} \cdot \nabla w_{\alpha_{2}}\right) w_{\alpha_{3}}-w_{\alpha_{1}} \times\left(w_{\alpha_{2}} \times H\left(w_{\alpha_{3}}\right)\right)\right) \\
& -\sum_{i=1}^{j-1} C_{j}^{i}\left(w_{i} \times \Delta w_{j-i}+w_{i} \times H\left(w_{j-i}\right)\right) \\
& +\sum_{i=0}^{j-1} C_{j}^{i}\left(\partial_{t}^{j-i} v \cdot \nabla\right) w_{i}+\sum_{\alpha \in \widetilde{A}_{j}} C^{\alpha} w_{\alpha_{1}} \times\left(\partial_{t}^{\alpha_{2}} v \cdot \nabla\right) w_{\alpha_{3}},
\end{array}
$$


with

$$
\bar{A}_{j}=\left\{\left(\alpha_{1}, \alpha_{2}, \alpha_{3}\right) \in\{0, \ldots, j-1\}^{3}, \alpha_{1}+\alpha_{2}+\alpha_{3}=j\right\}
$$

and

$$
\widetilde{A}_{j}=\left\{\left(\alpha_{1}, \alpha_{2}, \alpha_{3}\right) \in\{0, \ldots, j-1\} \times\{0, \ldots j\} \times\{0, \ldots, j-1\}, \alpha_{1}+\alpha_{2}+\alpha_{3}=j\right\} .
$$

In particular, $w_{k}=\partial_{t}^{k} w$ is in $L^{\infty}\left(0, T ; H^{1}(\Omega)\right) \cap L^{2}\left(0, T ; H^{2}(\Omega)\right)$ and satisfies:

$$
\begin{cases}\frac{\partial w_{k}}{\partial t}-\Delta w_{k}=-m \times \Delta w_{k}+K_{m}\left(\nabla w_{k}\right)+L_{m}\left(w_{k}\right)+F_{k}, \\ \frac{\partial w_{k}}{\partial \nu}=0 \quad \text { on } \mathbb{R}^{+} \times \partial \Omega, \\ w_{k}(t=0)=V_{k} .\end{cases}
$$

We aim to construct a more regular solution for (23). We establish before the following estimates.

Lemma 5.1. For all $j \in\{1, \ldots, k\}$, for all $T<T^{*}$,

$$
F_{j} \in L^{\infty}\left(0, T ; H^{2 k-2 j+1}(\Omega)\right) \cap L^{2}\left(0, T ; H^{2 k-2 j+2}(\Omega)\right) .
$$

Proof. We fix $j \leq k$. From Property $\mathcal{P}(k)$, for all $i \in\{0, \ldots, j-1\}, w_{i} \in L^{\infty}\left(0, T ; H^{2 k-2 j+3}(\Omega)\right) \cap$ $L^{2}\left(0, T ; H^{2 k-2 j+4}(\Omega)\right)$.

For all $\alpha \in \bar{A}_{j}, \nabla w_{\alpha_{1}}, \nabla w_{\alpha_{2}}, w_{\alpha_{1}}, w_{\alpha_{2}}, w_{\alpha_{3}}$ and $H\left(w_{\alpha_{3}}\right)$ are in $L^{\infty}\left(0, T ; H^{2 k-2 j+2}(\Omega)\right)$ which is an algebra since $2 k-2 j+2 \geq 2$. So

$$
\sum_{\alpha \in \bar{A}_{j}} C^{\alpha}\left(\left(\nabla w_{\alpha_{1}} \cdot \nabla w_{\alpha_{2}}\right) w_{\alpha_{3}}-w_{\alpha_{1}} \times\left(w_{\alpha_{2}} \times H\left(w_{\alpha_{3}}\right)\right)\right) \in L^{\infty}\left(0, T ; H^{2 k-2 j+2}(\Omega)\right),
$$

and $a$ fortiori this quantity is in $L^{\infty}\left(0, T ; H^{2 k-2 j+1}(\Omega)\right) \cap L^{2}\left(0, T ; H^{2 k-2 j+2}(\Omega)\right)$.

In the same way,

$$
\sum_{i=1}^{j-1} C_{j}^{i} w_{i} \times H\left(w_{j-i}\right) \in L^{\infty}\left(0, T ; H^{2 k-2 j+1}(\Omega)\right) \cap L^{2}\left(0, T ; H^{2 k-2 j+2}(\Omega)\right) .
$$

On the other hand, for $i \in\{1, \ldots, j-1\}, w_{i} \in L^{\infty}\left(0, T ; H^{2 k-2 j+2}(\Omega)\right)$ and $\Delta w_{j-i} \in L^{\infty}\left(0, T ; H^{2 k-2 j+1}(\Omega) \cap\right.$ $L^{2}\left(0, T ; H^{2 k-2 j+2}(\Omega)\right)$. So, since $H^{s}(\Omega)$ is an algebre for $s \geq 2$ or by applying Lemma 2.3 , we obtain that:

$$
\sum_{i=1}^{j-1} C_{j}^{i} w_{i} \times \Delta w_{j-i} \in L^{\infty}\left(0, T ; H^{2 k-2 j+1}(\Omega)\right) \cap L^{2}\left(0, T ; H^{2 k-2 j+2}(\Omega)\right) .
$$

Since for all $i \leq j, \partial_{t}^{i} v \in \mathcal{C}^{0}\left(0, T ; H^{2 k-2 j+1}(\Omega)\right) \cap L^{2}\left(0, T ; H^{2 k-2 j+2}(\Omega)\right)$, with the same arguments as below, we obtain that the remainder terms are in $L^{\infty}\left(0, T ; H^{2 k-2 j+1}(\Omega)\right) \cap L^{2}\left(0, T ; H^{2 k-2 j+2}(\Omega)\right)$.

\subsection{Construction of more regular solution for (24)}

We consider the following Cauchy problem:

$$
\begin{cases}\frac{\partial w}{\partial t}-\Delta w=-m \times \Delta w+K_{m}(\nabla w)+L_{m}(w)+F_{k} \\ \frac{\partial w}{\partial \nu}=0 \quad \text { on } \mathbb{R}^{+} \times \partial \Omega \\ w(t=0)=V_{k} .\end{cases}
$$


We aim to construct a solution for $(24)$ in $L^{\infty}\left(0, T ; H^{2}(\Omega)\right) \cap L^{2}\left(0, T ; H^{3}(\Omega)\right)$

We recall that, from the compatibility condition at order $k, V_{k} \in H^{2}(\Omega)$ and $\frac{\partial V_{k}}{\partial \nu}=0$ on $\partial \Omega$.

We consider the following Galerkin approximation for (24):

$$
\left\{\begin{array}{l}
w^{n} \in \mathcal{C}^{1}\left(\left[0, T_{n}\left[; V_{n}\right)\right.\right. \\
\frac{\partial w^{n}}{\partial t}-\Delta w^{n}=P_{n}\left(-m \times \Delta w^{n}+K_{m}\left(\nabla w^{n}\right)+L_{m}\left(w^{n}\right)+F_{k}\right), \\
w^{n}(t=0)=P_{n}\left(V_{k}\right) .
\end{array}\right.
$$

Since the coefficients of this ordinary differential equation are continuous on $\left[0, T^{*}[\right.$, since the equation is linear, the maximal existence time $T_{n}$ equals $T^{*}$. Let us obtain uniform estimates on $w^{n}$.

Bound for the initial data. Using the compatibility condition at order $k$, we know that $V_{k} \in$ $H^{2}(\Omega)$ and that $\frac{\partial V_{k}}{\partial \nu}=0$ on $\partial \Omega$, so we can apply Proposition 1:

$$
\exists K, \quad \forall n,\left\|w^{n}(0)\right\|_{H^{2}} \leq K .
$$

$L^{2}$ estimate. We multiply (25) by $w^{n}$ and we obtain that:

where

$$
\frac{1}{2} \frac{d}{d t}\left\|w^{n}\right\|_{L^{2}}^{2}+\left\|\nabla w^{n}\right\|_{L^{2}}^{2}=T_{1}+T_{2}+T_{3}+T_{4}
$$

$$
\begin{aligned}
T_{1} & =-\int_{\Omega} m \times \Delta w^{n} \cdot w^{n}=\int_{\Omega} m \times w^{n} \cdot \Delta w_{n} \\
& =-\int_{\Omega} \nabla\left(m \times w^{n}\right) \cdot \nabla w^{n} \quad \text { (using the homogeneous boundary conditions) } \\
& =\int_{\Omega}(\nabla m) \times w^{n} \cdot \nabla w^{n} \\
\leq & \|\nabla m\|_{L^{\infty}}\left\|w^{n}\right\|_{L^{2}}\left\|\nabla w^{n}\right\|_{L^{2}}, \\
T_{2}= & \int_{\Omega} K_{m}\left(\nabla w^{n}\right) w^{n} \\
\leq & \left\|K_{m}\left(\nabla w^{n}\right)\right\|_{L^{2}}\left\|w^{n}\right\|_{L^{2}} \\
\leq & \left(2\|\nabla m\|_{L^{\infty}}+2\|v\|_{L^{\infty}}\right)\left\|w^{n}\right\|_{L^{2}}\left\|\nabla w^{n}\right\|_{L^{2}} \quad\left(\text { since }\|m\|_{L^{\infty}}=1\right), \\
T_{3}= & \int_{\Omega} L_{m}\left(w^{n}\right) w^{n} \\
\leq & \left\|L_{m}\left(w^{n}\right)\right\|_{L^{2}}\left\|w^{n}\right\|_{L^{2}} \\
\leq & \left(\|\nabla m\|_{L^{6}}^{2}+\|\Delta m\|_{L^{3}}+\|\nabla m\|_{L^{6}}\|v\|_{L^{\infty}}\right)\left\|w^{n}\right\|_{L^{6}}\left\|w^{n}\right\|_{L^{2}} \\
& +C\left(\|H(m)\|_{L^{\infty}}+1\right)\left\|w^{n}\right\|_{L^{2}}^{2}, \\
T_{4}= & \int_{\Omega} F_{k} \cdot w^{n} \\
\leq & \left\|F_{k}\right\|_{L^{2}}\left\|w^{n}\right\|_{L^{2}} \cdot \\
& \\
&
\end{aligned}
$$


$H^{2}$ estimate. We multiply (25) by $-\Delta^{2} w^{n}$ and we obtain that:

$$
\frac{1}{2} \frac{d}{d t}\left\|\Delta w^{n}\right\|_{L^{2}}^{2}+\left\|\nabla \Delta w^{n}\right\|_{L^{2}}^{2} \leq I_{1}+I_{2}+I_{3}+I_{4}
$$

where

$$
\begin{aligned}
& I_{1}=\left\|(\nabla m) \times \Delta w^{n}\right\|_{L^{2}}\left\|\nabla \Delta w^{n}\right\|_{L^{2}} \\
& \leq\|\nabla m\|_{L^{\infty}}\left\|w^{n}\right\|_{L^{2}}\left\|\nabla \Delta w^{n}\right\|_{L^{2}}, \\
& I_{2}=\left|\int_{\Omega} \nabla\left(K_{m}\left(\nabla w^{n}\right)\right) \nabla \Delta w^{n}\right| \\
& \leq C \int_{\Omega}\left(\left|D^{2} m\right|\left|\nabla w^{n}\right|+|\nabla m|\left|D^{2} w^{n}\right|+|\nabla m|^{2}\left|\nabla w^{n}\right|+|\nabla v|\left|\nabla w^{n}\right|\right)\left|\nabla \Delta w^{n}\right| \\
& +\int_{\Omega}\left(|v|\left|D^{2} w^{n}\right|+|\nabla m||v|\left|\nabla w^{n}\right|\right)\left|\nabla \Delta w^{n}\right| \\
& \leq C\left(\left\|D^{2} m\right\|_{L^{3}}\left\|\nabla w^{n}\right\|_{L^{6}}+\|\nabla m\|_{L^{\infty}}\left\|D^{2} w^{n}\right\|_{L^{2}}+\|\nabla m\|_{L^{6}}^{2}\left\|\nabla w^{n}\right\|_{L^{6}}\right. \\
& \left.+\|\nabla v\|_{L^{\infty}}\left\|\nabla w^{n}\right\|_{L^{2}}+\|v\|_{L^{\infty}}\left\|D^{2} w^{n}\right\|_{L^{2}}+\|\nabla m\|_{L^{\infty}}\|v\|_{L^{\infty}}\left\|\nabla w^{n}\right\|_{L^{2}}\right)\left\|\nabla \Delta w^{n}\right\|_{L^{2}}, \\
& I_{3}=\left|\int_{\Omega} \nabla\left(L_{m}\left(w^{n}\right)\right) \cdot \nabla \Delta w^{n}\right| \\
& \leq C \int_{\Omega}\left(\left|D^{2} m\right|\left|\nabla w^{n}\right|+|\nabla m|^{2}\left|\nabla w^{n}\right|+|\Delta m|\left|\nabla w^{n}\right|+|\nabla \Delta m|\left|w^{n}\right|+\left|w^{n}\right||\nabla H(m)|\right)\left|\nabla \Delta w^{n}\right| \\
& +C \int_{\Omega}\left(|H(m)|\left|\nabla w^{n}\right|+|\nabla m|\left|w^{n}\right|+\left|\nabla w^{n}\right||v||\nabla m|+\left|w^{n}\right||\nabla v||\nabla m|+\left|w^{n}\right||v|\left|D^{2} m\right|\right)\left|\nabla \Delta w^{n}\right| \\
& \leq C\left(\left\|D^{2} m\right\|_{L^{3}}+\|\nabla m\|_{L^{6}}^{2}+\|H(m)\|_{L^{3}}+\|\nabla m\|_{L^{3}}+\|v\|_{L^{\infty}}\right)\left\|\nabla w^{n}\right\|_{L^{6}}\left\|\nabla \Delta w^{n}\right\|_{L^{2}} \\
& +C\left(\|\nabla \Delta m\|_{L^{2}}+\|\nabla H(m)\|_{L^{2}}+\|\nabla m\|_{L^{2}}+\|\nabla v\|_{L^{4}}\|\nabla m\|_{L^{4}}\right)\left\|w^{n}\right\|_{L^{\infty}}\left\|\nabla \Delta w^{n}\right\|_{L^{2}} \\
& +\|v\|_{L^{\infty}}\left\|D^{2} m\right\|_{L^{4}}\left\|w^{n}\right\|_{L^{\infty}}\left\|\nabla \Delta w^{n}\right\|_{L^{2}}, \\
& I_{4} \leq\left\|\nabla F_{k}\right\|_{L^{2}}\left\|\nabla \Delta w^{n}\right\|_{L^{2}} .
\end{aligned}
$$

By adding up the previous $L^{2}$ and $H^{2}$ estimates, after absorbing $\left\|\nabla \Delta w^{n}\right\|_{L^{2}}$, we obtain that

$$
\frac{d}{d t}\left\|w^{n}\right\|_{2, \Omega}^{2}+\left\|\nabla w^{n}\right\|_{2, \Omega}^{2} \leq g_{1}(t)\left\|w^{n}\right\|_{2, \Omega}^{2}+g_{2}^{n}(t)
$$

where

$$
\begin{aligned}
& g_{1}(t)=C\left(1+\|m\|_{H^{2}}^{2}\right)\left(1+\|v\|_{H^{2}}\right)+C\|m\|_{H^{3}}^{2}, \\
& g_{2}^{n}(t)=C\left\|F_{k}\right\|_{H^{1}}^{2} .
\end{aligned}
$$

We already know that for all $T<T^{*}, m \in L^{\infty}\left(0, T ; H^{2}(\Omega)\right) \cap L^{2}\left(0, T ; H^{3}(\Omega)\right)$, so $g_{1} \in L^{1}(0, T)$ for all $T<T^{*}$. In addition, by lemma 5.1, $g_{2}^{n}$ is uniformly bounded (with respect to $n$ ) in $L^{1}(0, T)$ for all $T<T^{*}$. Since (26) yields a uniform bound for $\left\|w^{n}(0)\right\|_{2, \Omega}^{2}$, by the comparison lemma, we deduce that for all $T<T^{*}$, there exists a constant $C(T)$ such that for all $n$,

$$
\left\|w^{n}\right\|_{L^{\infty}\left(0, T ; H^{2}(\Omega)\right)}+\left\|w^{n}\right\|_{L^{2}\left(0, T ; H^{3}(\Omega)\right)} \leq C(T) .
$$


By the equation (25), we obtain in addition the following estimate on the time derivatives:

$$
\forall T<T^{*}, \quad \exists C(T), \quad \forall n, \quad\left\|\partial_{t} w^{n}\right\|_{L^{\infty}\left(0, T ; L^{2}(\Omega)\right)}+\left\|\partial_{t} w^{n}\right\|_{L^{2}\left(0, T ; H^{1}(\Omega)\right)} \leq C(T) .
$$

By standard arguments (see section 3.3) we obtain by extracting subsequences that there exists $w$ satisfying (24) such that

$$
\forall T<T^{*}, \quad w \in L^{\infty}\left(0, T ; H^{2}(\Omega)\right) \cap L^{2}\left(0, T ; H^{3}(\Omega)\right) .
$$

\subsection{Uniqueness for (24)}

Let $w_{1}$ and $w_{2}$ two solutions of $(24)$ in $L^{\infty}\left(0, T ; H^{1}(\Omega)\right) \cap L^{2}\left(0, T ; H^{2}(\Omega)\right)$. We denote $W=w_{1}-w_{2}$, and we remark that $w$ satisfies the following problem:

$$
\begin{cases}\frac{\partial W}{\partial t}-\Delta W=-m \times \Delta W+K_{m}(\nabla W)+L_{m}(W), & \text { on } \mathbb{R}^{+} \times \partial \Omega, \\ \frac{\partial W}{\partial \nu}=0 & \\ W(t=0)=0 . & \end{cases}
$$

Since $W \in L^{\infty}\left(0, T ; H^{1}(\Omega)\right) \cap L^{2}\left(0, T ; H^{2}(\Omega)\right)$ and since $\partial_{t} W \in L^{2}\left(0, T ; L^{2}(\Omega)\right)$, we can perform the following $L^{2}$ estimate, taking the inner product of the equation with $W$ :

$$
\begin{aligned}
\frac{d}{d t}\|W\|_{L^{2}}^{2}+2\|\nabla W\|_{L^{2}}^{2} \leq & C\left(\|\nabla m\|_{L^{\infty}}+\|v\|_{L^{\infty}}\right)\|W\|_{L^{2}}\|\nabla W\|_{L^{2}} \\
& +\left(\|\nabla m\|_{L^{6}}^{2}+\|\Delta m\|_{L^{3}}+\|\nabla m\|_{L^{6}}\|v\|_{L^{\infty}}\right)\|W\|_{L^{6}}\|W\|_{L^{2}} \\
& +C\left(\|H(m)\|_{L^{\infty}}+1\right)\|W\|_{L^{2}}^{2} .
\end{aligned}
$$

We multiply Equation (30) by $-\Delta W$ and we integrate on $[0, T] \times \Omega$ for all $T<T^{*}$. We can do it because $\Delta W$ and $\frac{\partial W}{\partial t}$ are in $L^{2}([0, T] \times \Omega)$. Using that $W \in \mathcal{C}^{0}\left(0, T ; H^{1}(\Omega)\right)$ and that $W(0)=0$, we obtain that:

We have

$$
\|\nabla W(T)\|_{L^{2}}^{2}+2 \int_{0}^{T} \int_{\Omega}|\Delta W|^{2} d x d t=-2 \int_{0}^{T} \int_{\Omega}\left(K_{m}(\nabla W)+L_{m}(W)\right) \Delta W
$$

and

$$
\left\|K_{m}(\nabla W)\right\|_{L^{2}} \leq C\|\nabla m\|_{L^{\infty}}\|\nabla W\|_{L^{2}}+C\|v\|_{L^{\infty}}\|W\|_{L^{2}},
$$

$$
\left\|L_{m}(W)\right\|_{L^{2}} \leq C\|\nabla m\|_{L^{6}}^{2}\|W\|_{L^{6}}+C\|W\|_{L^{6}}\|\Delta m\|_{L^{3}}+C\|W\|_{L^{2}}\left(\|H(m)\|_{L^{\infty}}+\|\nabla m\|_{L^{\infty}}\|v\|_{L^{\infty}}\right) .
$$

We integrate the $L^{2}$ estimate from 0 to $T$. Adding up with the $H^{1}$ estimate and using Young formula, we obtain that:

$\|W(T)\|_{L^{2}}^{2}+\|\nabla W(T)\|_{L^{2}}^{2}+\int_{0}^{T} \int_{\Omega}\left(|\nabla W|^{2}+|\Delta W|^{2}\right) d x d t \leq C \int_{0}^{T}\left(\|\nabla W(t)\|_{L^{2}}^{2}+\|W(t)\|_{L^{2}}^{2}\right) g(t) d t$,

where

$$
g(t)=1+\|v(t)\|_{L^{\infty}}^{2}+\|m(t)\|_{2, \Omega}^{4}+\|\nabla m(t)\|_{L^{\infty}}^{2}\left(1+\|v(t)\|_{L^{\infty}}^{2}\right) .
$$

By properties of $m$ and $v, g \in L^{1}(0, T)$ for all $T<T^{*}$ so that we can use the Gronwall lemma to conclude that

$$
W=0 \text { on }\left[0, T^{*}[\times \Omega .\right.
$$

Now, $w$ and $\partial_{t}^{k} m$ are solutions for $(24)$ in the space $\mathcal{C}^{0}\left(\left[0, T^{*}\left[; H^{1}(\Omega)\right) \cap L^{2}\left(0, T ; H^{2}(\Omega)\right)\right.\right.$, so by the previous uniqueness result, $w=\partial_{t}^{k} m$, so that

$$
\forall T<T^{*}, \quad \partial_{t}^{k} m \in L^{\infty}\left(0, T ; H^{2}(\Omega)\right) \cap L^{2}\left(0, T ; H^{3}(\Omega)\right) .
$$




\subsection{Regularity for $m$}

From Property $\mathcal{P}(k)$ we know that

$$
\forall j \in\{0, \ldots, k\}, \quad w_{j}:=\partial_{t}^{j} m \in L^{\infty}\left(0, T ; H^{2 k-2 j+1}(\Omega)\right) \cap L^{2}\left(0, T ; H^{2 k-2 j+2}(\Omega)\right) .
$$

In addition, we have proven in the previous subsection that $\partial_{t}^{k} m \in L^{\infty}\left(0, T ; H^{2}(\Omega)\right) \cap L^{2}\left(0, T ; H^{3}(\Omega)\right)$.

Let us prove by induction the following claim:

Claim. For all $T<T^{*}$, for $j \in\{0, \ldots k-1\}, w_{k-j} \in L^{\infty}\left(0, T ; H^{2+2 j}(\Omega)\right) \cap L^{2}\left(0, T ; H^{3+2 j}(\Omega)\right)$.

Proof. This property is true for $j=0$ by (31).

Let $j \in\{1, \ldots, k-1\}$. Let us assume that the property is true at the rank $j-1$. Then, from Equation (22), replacing $j$ by $k-j$, we have:

$$
\Delta w_{k-j}-m \times \Delta w_{k-j}=\partial_{t} w_{k-j}-K_{m}\left(\nabla w_{k-j}\right)-L_{m}\left(w_{k-j}\right)-F_{k-j},
$$

that is, since $\partial_{t} w_{k-j}=w_{k-j+1}$,

$$
\Delta w_{k-j}=\psi_{m}\left(w_{k-j+1}-K_{m}\left(\nabla w_{k-j}\right)-L_{m}\left(w_{k-j}\right)-F_{k-j}\right)
$$

(where $\psi_{m}$ is defined on page 16). We have:

- $m \in L^{\infty}\left(0, T ; H^{2 k+1}(\Omega)\right)$

- $\nabla m \in L^{\infty}\left(0, T ; H^{2 k}(\Omega)\right)$

- $\Delta m \in L^{\infty}\left(0, T ; H^{2 k-1}(\Omega)\right)$

- $w_{k-j} \in L^{\infty}\left(0, T ; H^{2 j+1}(\Omega)\right)$

- $v \in L^{\infty}\left(0, T ; H^{2 k}(\Omega)\right)$.

On the one hand, we recall that

$$
K_{m}\left(\nabla w_{k-j}\right)=2\left(\nabla m \cdot \nabla w_{k-j}\right) m+(v \cdot \nabla) w_{k-j}+m \times(v \cdot \nabla) w_{k-j}
$$

Since $2 j \leq 2 k, \nabla m, \nabla w_{k-j}, v$ and $m$ are in $L^{\infty}\left(0, T ; H^{2 j}(\Omega)\right)$, which is an algebra (since $\left.j \geq 1\right)$, then

$$
K_{m}\left(\nabla w_{k-j}\right) \in L^{\infty}\left(0, T ; H^{2 j}(\Omega)\right)
$$

Since $2 j+1 \leq 2 k, \nabla m, v$ and $m$ are in $L^{\infty}\left(0, T ; H^{2 j+1}(\Omega)\right)$ and since $\nabla w_{k-j} \in L^{2}\left(0, T ; H^{2 j+1}(\Omega)\right)$, then

$$
K_{m}\left(\nabla w_{k-j}\right) \in L^{2}\left(0, T ; H^{2 j+1}(\Omega)\right) .
$$

On the other hand, we recall that

$$
\begin{aligned}
L_{m}\left(w_{k-j}\right)= & |\nabla m|^{2} w_{k-j}-w_{k-j} \times \Delta m-w_{k-j} \times H(m)-m \times H\left(w_{k-j}\right)-w_{k-j} \times(m \times H(m)) \\
& -m \times\left(w_{k-j} \times H(m)\right)-m \times\left(m \times H\left(w_{k-j}\right)\right)+w_{k-j} \times(v \cdot \nabla) m
\end{aligned}
$$

From Proposition $2, H\left(w_{k-j}\right)$ and $w_{k-j}$ have the same regularity, i.e. are in $L^{\infty}\left(0, T ; H^{2 j+1}(\Omega)\right)$. We remark that $j \leq k-1$ so that $2 j+1 \leq 2 k-1$. So $\nabla m, H(m), m$ and $\Delta m$ are in $L^{\infty}\left(0, T ; H^{2 j+1}(\Omega)\right)$. The same holds for $v$. Since this space is an algebra, we obtain that

$$
L_{m}\left(w_{k-j}\right) \in L^{\infty}\left(0, T ; H^{2 j+1}(\Omega)\right) .
$$

From Lemma 5.1,

$$
F_{k-j} \in L^{\infty}\left(0, T ; H^{2 j+1}(\Omega)\right) .
$$


From the property at rank $j-1$,

$$
w_{k-j+1} \in L^{\infty}\left(0, T ; H^{2 j}(\Omega)\right) \cap L^{2}\left(0, T ; H^{2 j+1}(\Omega)\right) .
$$

In addition, since $m \in L^{\infty}\left(0, T ; H^{2 j+1}(\Omega)\right)$, we keep the same regularity when we compose with $\psi_{m}$, so:

$$
\Delta w_{k-j} \in L^{\infty}\left(0, T ; H^{2 j}(\Omega)\right) \cap L^{2}\left(0, T ; H^{2 j+1}(\Omega)\right),
$$

and by elliptic regularity results,

$$
w_{k-j} \in L^{\infty}\left(0, T ; H^{2 j+2}(\Omega)\right) \cap L^{2}\left(0, T ; H^{2 j+3}(\Omega)\right) .
$$

This concludes the proof of the claim.

In particular, we have proven that

$$
w_{1}=\partial_{t} m \in L^{\infty}\left(0, T ; H^{2 k}(\Omega)\right) \cap L^{2}\left(0, T ; H^{2 k+1}(\Omega)\right) .
$$

Now, we have:

$$
\Delta m=\frac{1}{2}\left(\partial_{t} m+m \times \partial_{t} m\right)-|\nabla m|^{2} m+W,
$$

where $W=m \times(m \times H(m))-m \times(v \cdot \nabla) m$.

Since $\nabla m, m$ and $H(m)$ are in $L^{\infty}\left(0, T ; H^{2 k}(\Omega)\right)$, the same holds for $-|\nabla m|^{2} m+W$.

Since $m \in L^{\infty}\left(0, T ; H^{2 k+1}(\Omega)\right), \partial_{t} m+m \times \partial_{t} m \in L^{\infty}\left(0, T ; H^{2 k}(\Omega)\right) \cap L^{2}\left(0, T ; H^{2 k+1}(\Omega)\right)$.

Therefore, $\Delta m \in L^{\infty}\left(0, T ; H^{2 k}(\Omega)\right) \cap L^{2}\left(0, T ; H^{2 k+1}(\Omega)\right)$, so

$$
m \in L^{\infty}\left(0, T ; H^{2 k+2}(\Omega)\right) \cap L^{2}\left(0, T ; H^{2 k+3}(\Omega)\right) .
$$

\subsection{Regularity for $\partial_{t}^{k} m$ in the case $m_{0} \in H^{2 k+3}(\Omega)$}

We assume now in addition that $m_{0} \in H^{2 k+3}\left(\Omega ; S^{2}\right)$ and satisfies the compatibility condition at order $k$. From the previous case, we already know that

$$
\forall j \in\{0, \ldots k\}, \forall T<T^{*}, \partial_{t}^{j} m \in L^{\infty}\left(0, T ; H^{2 k+2-2 j}(\Omega)\right) \cap L^{2}\left(0, T ; H^{2 k+3-2 j}(\Omega)\right) .
$$

In addition, we know that $\partial_{t}^{k} m$ is the unique solution for Equation (24). We will prove that we can improve the regularity of $\partial_{t}^{k} m$ using the Galerkin formulation (25).

We derivate (25) with respect to time. Denoting by $\alpha_{n}:=\partial_{t} w^{n}$, we obtain that:

$$
\left\{\begin{array}{l}
\frac{\partial \alpha^{n}}{\partial t}-\Delta \alpha^{n}=-P_{n}\left(m \times \Delta \alpha^{n}+K_{m}\left(\nabla \alpha^{n}\right)+L_{m}\left(\alpha^{n}\right)+\widetilde{F}_{k}\right) \text { on }\left[0, T_{n}[,\right. \\
\alpha^{n}(0)=P_{n}\left(\Delta\left(P_{n}\left(V_{k}\right)\right)-m_{0} \times \Delta\left(P_{n}\left(V_{k}\right)\right)+K_{m_{0}}\left(\nabla\left(P_{n}\left(V_{k}\right)\right)\right)+L_{m_{0}}\left(P_{n}\left(V_{k}\right)\right)+F_{k}(t=0)\right),
\end{array}\right.
$$

where

$$
\widetilde{F}_{k}=-\partial_{t} m \times \Delta w^{n}+\partial_{t}\left(K_{m}\right)\left(\nabla w^{n}\right)+\partial_{t}\left(L_{m}\right)\left(w^{n}\right)+\partial_{t} F_{k} .
$$

We multiply (32) by $\Delta \alpha^{n}$. After integration on $\Omega$ and using Young formula, we obtain that:

$$
\frac{d}{d t}\left\|\nabla \alpha^{n}\right\|_{L^{2}}^{2}+\left\|\Delta \alpha^{n}\right\|_{L^{2}}^{2} \leq C\left(\left\|K_{m}\left(\nabla \alpha^{n}\right)\right\|_{L^{2}}^{2}+\left\|L_{m}\left(\alpha^{n}\right)\right\|_{L^{2}}^{2}+\left\|\widetilde{F}_{k}\right\|_{L^{2}}^{2}\right) .
$$


We have:

$$
\begin{aligned}
\left\|K_{m}\left(\nabla \alpha^{n}\right)\right\|_{L^{2}}= & \left\|2\left(\nabla m \cdot \nabla \alpha^{n}\right) m+(v \cdot \nabla) \alpha^{n}+m \times(v \cdot \nabla) \alpha^{n}\right\|_{L^{2}} \\
\leq & C\left(\|\nabla m\|_{L^{\infty}}+\|v\|_{L^{\infty}}\right)\left\|\nabla \alpha^{n}\right\|_{L^{2}}, \\
\left\|L_{m}\left(\alpha^{n}\right)\right\|_{L^{2}} \leq & \left\||\nabla m|^{2} \alpha^{n}-\alpha^{n} \times \Delta m-\alpha^{n} \times H(m)-m \times H\left(\alpha^{n}\right)-\alpha^{n} \times(m \times H(m))\right\|_{L^{2}} \\
& +\left\|m \times\left(\alpha^{n} \times H(m)\right)+m \times\left(m \times H\left(\alpha^{n}\right)\right)\right\|_{L^{2}}+\left\|\alpha^{n} \times(v \cdot \nabla) m\right\|_{L^{2}} \\
\leq & C\left(\|\nabla m\|_{L^{\infty}}^{2}+\|\Delta m\|_{L^{\infty}}+\|H(m)\|_{L^{\infty}}+\|v\|_{L^{\infty}}\|\nabla m\|_{L^{\infty}}+1\right)\left\|\alpha^{n}\right\|_{L^{2}} \\
\leq & C(T) \text { for all } T<T^{*},
\end{aligned}
$$

since $m \in L^{\infty}\left(0, T ; H^{4}(\Omega)\right)$ and by $(29)$.

Concerning $\widetilde{F}_{k}$, we have:

$$
\begin{aligned}
\left\|\partial_{t} m \times \Delta w^{n}\right\|_{L^{2}} \leq & C\left\|\partial_{t} m\right\|_{L^{\infty}}\left\|w^{n}\right\|_{H^{2}} \\
& \leq C(T) \text { for all } T<T^{*},
\end{aligned}
$$

since $\partial_{t} m \in L^{\infty}\left(0, T ; H^{2 k}(\Omega)\right)$ and by $(28)$,

$$
\begin{aligned}
\partial_{t}\left(K_{m}\right)\left(\nabla w^{k}\right)= & 2\left(\nabla \partial_{t} m \cdot \nabla w^{n}\right) m+2\left(\nabla m \cdot \nabla w^{n}\right) \partial_{t} m+\left(\partial_{t} v \cdot \nabla\right) w^{n}+\partial_{t} m \times(v \cdot \nabla) w^{n} \\
& +m \times\left(\partial_{t} v \cdot \nabla\right) w^{n} \\
\left\|\partial_{t}\left(K_{m}\right)\left(\nabla w^{k}\right)\right\|_{L^{2}} \leq & C\left\|\nabla w^{n}\right\|_{L^{6}}\left(\left\|\nabla \partial_{t} m\right\|_{L^{3}}+\|\nabla m\|_{L^{\infty}}\left\|\partial_{t} m\right\|_{L^{3}}+\left\|\partial_{t} v\right\|_{L^{3}}+\|v\|_{L^{\infty}}\left\|\partial_{t} m\right\|_{L^{3}}\right) .
\end{aligned}
$$

Since $k \geq 1$, since $\partial_{t} m \in L^{\infty}\left(0, T ; H^{2}(\Omega)\right)$, then $\nabla \partial_{t} m \in L^{\infty}\left(0, T ; H^{1}(\Omega)\right)$ so by embedding, for all $t \leq T<T^{*},\left\|\nabla \partial_{t} m(t)\right\|_{L^{3}} \leq K(T)$.

In addition,

$$
\left\|\nabla w^{n}\right\|_{L^{6}} \leq C\left\|w^{n}\right\|_{H^{2}} \leq K(T) \text { by }(28) .
$$

So,

$$
\begin{aligned}
& \left\|\partial_{t}\left(K_{m}\right)\left(\nabla w^{k}\right)(t)\right\|_{L^{2}} \leq K(T) \text { for all } t \leq T<T^{*} . \\
\partial_{t}\left(L_{m}\right)\left(w_{k}\right)= & 2\left(\nabla m \cdot \nabla \partial_{t} m\right) w^{n}-w^{n} \times \Delta \partial_{t} m-w^{n} \times H\left(\partial_{t} m\right)-\partial_{t} m \times H\left(w^{n}\right) \\
& -w^{n} \times\left(\partial_{t} m \times H(m)\right)-w^{n} \times\left(m \times H\left(\partial_{t} m\right)\right)-\partial_{t} m \times\left(w^{n} \times H(m)\right) \\
& -m \times\left(w^{n} \times H\left(\partial_{t} m\right)\right)-\partial_{t} m \times\left(m \times H\left(w^{n}\right)\right)-m \times\left(\partial_{t} m \times H\left(w^{n}\right)\right) \\
& +w^{n} \times\left(\partial_{t} v \cdot \nabla\right) m+w^{n} \times(v \cdot \nabla) \partial_{t} m \\
\left\|\partial_{t}\left(L_{m}\right)\left(w_{k}\right)\right\|_{L^{2}} \leq & C\left\|w^{n}\right\|_{L^{\infty}}\left(\|\nabla m\|_{L^{\infty}}\left\|\nabla \partial_{t} m\right\|_{L^{2}}+\left\|\Delta \partial_{t} m\right\|_{L^{2}}+\left\|H\left(\partial_{t} m\right)\right\|_{L^{2}}\right) \\
& +C\left\|w^{n}\right\|_{L^{\infty}}\left(\left\|\partial_{t} m\right\|_{L^{2}}\|H(m)\|_{L^{\infty}}+\left\|\partial_{t} m\right\|_{L^{2}}+\|v\|_{L^{\infty}}\left\|\nabla \partial_{t} m\right\|_{L^{2}}\right) \\
& +C\left\|w^{n}\right\|_{L^{\infty}}\left\|\partial_{t} v\right\|_{L^{\infty}}\|\nabla m\|_{L^{2}}+C\left\|H\left(w^{n}\right)\right\|_{L^{2}}\left\|\partial_{t} m\right\|_{L^{2}} .
\end{aligned}
$$

Using Proposition 2, using (28) and the known bounds on $\partial_{t} m$, we obtain that:

$$
\forall T<T^{*}, \exists C(T), \forall n, \frac{d}{d t}\left\|\alpha^{n}\right\|_{L^{2}}^{2}+\left\|\Delta \alpha^{n}\right\|_{L^{2}}^{2} \leq C(T)\left\|\nabla \alpha^{n}\right\|_{L^{2}}^{2}+C(T) .
$$


Now from Proposition 1, we have:

$$
\begin{aligned}
\left\|\nabla \alpha^{n}(0)\right\|_{L^{2}} & \leq\left\|\Delta\left(P_{n}\left(V_{k}\right)\right)-m_{0} \times \Delta\left(P_{n}\left(V_{k}\right)\right)+K_{m_{0}}\left(\nabla\left(P_{n}\left(V_{k}\right)\right)\right)+L_{m_{0}}\left(P_{n}\left(V_{k}\right)\right)+F_{k}(t=0)\right\|_{H^{1}} \\
& \leq C\left(1+\left\|P_{n}\left(V^{k}\right)\right\|_{H^{3}}\right) .
\end{aligned}
$$

From the compatibility condition at order $k, \frac{\partial V^{k}}{\partial \nu}=0$ on $\partial \Omega$, so from Proposition 1,

$$
\left\|P_{n}\left(V^{k}\right)\right\|_{H^{3}} \leq C\left\|V^{k}\right\|_{H^{3}} \text { for all } n .
$$

Therefore, there exists a constant $C$ such that for all $n$,

$$
\left\|\nabla \alpha^{n}(0)\right\|_{L^{2}} \leq C .
$$

Estimates (33) and (34) coupled with the comparison lemma 2.4 yield that:

$$
\forall T<T^{*}, \exists C(T),\left\|\alpha^{n}\right\|_{L^{\infty}\left(0, T ; H^{1}(\Omega)\right)}+\left\|\alpha^{n}\right\|_{L^{2}\left(0, T ; H^{2}(\Omega)\right)} \leq C(T) .
$$

Since $\alpha^{n} \rightarrow \partial_{t}^{k+1} m$ in the distribution sense, we obtain that

$$
\forall T<T^{*}, \quad \partial_{t}^{k+1} m \in L^{\infty}\left(0, T ; H^{1}(\Omega)\right) \cap L^{2}\left(0, T ; H^{2}(\Omega)\right) .
$$

\subsection{Regularity for $m$ in the case $m_{0} \in H^{2 k+3}(\Omega)$}

We know from Section 5.3 that

$$
\forall j \in\{0, \ldots, k\}, \quad \partial_{t}^{j} m \in L^{\infty}\left(0, T ; H^{2 k-2 j+2}(\Omega)\right) \cap L^{2}\left(0, T ; H^{2 k-2 j+3}(\Omega)\right) .
$$

So coupling this estimate with (35), we gain one rank for the regularity of each $w_{j}$, and exactly with the same method as in Section 5.3, we prove that

$$
\forall j \in\{0, \ldots, k+1\}, \quad \partial_{t}^{j} m \in L^{\infty}\left(0, T ; H^{2 k-2 j+3}(\Omega)\right) \cap L^{2}\left(0, T ; H^{2 k-2 j+4}(\Omega)\right),
$$

so that the proof of Property $\mathcal{P}(k+1)$ is complete.

\section{References}

[1] François Alouges and Alain Soyeur, On global weak solutions for Landau-Lifshitz equations: existence and nonuniqueness, Nonlinear Anal. 18 (1992), no. 11, 1071-1084.

[2] T. Aubin, Un théorème de compacité, C. R. Acad. Sci. Paris 256 (1963), 5242-5044.

[3] Gaël Bonithon, Landau-Lifschitz-Gilbert equation with applied electric current. Discrete Contin. Dyn. Syst. 2007, Dynamical Systems and Differential Equations. Proceedings of the 6th AIMS International Conference, suppl., 138-144.

[4] F. Boyer, P. Fabrie, Eléments d'analyse pour l'étude de quelques modèles d'écoulements de fluides visqueux incompressibles, Mathématiques \& Applications (Berlin) 52. Springer-Verlag, Berlin, 2006.

[5] William F. Brown, Micromagnetics, Wiley, New York (1963).

[6] Gilles Carbou and Pierre Fabrie, Time average in micromagnetism, J. Differential Equations 147 (1998), no. 2, 383-409.

[7] G. Carbou, P. Fabrie, Regular Solutions for Landau-Lifschitz equations in a bounded domain, Differential Integral Equations 14 (2001), 213-229. 
[8] G. Carbou, P. Fabrie, O. Guès, On the ferromagnetism equations in the non static case, Commun. Pure Appl. Anal. 3 (2004), no. 3, 367-393.

[9] G. Foias and R. Temam, Remarques sur les équations de Navier-Stokes stationnaires et les phénomènes successifs de bifurcation, An. Sc. Norm. Super. Pisa IV, 5 (1978), 29-63.

[10] O.A. ladyzhenskaya, The boundary value problem of mathematical physic, Springer Verlag Applied Math. Sciences, 49, 1985.

[11] L. Landau et E. Lifschitz, Electrodynamique des milieux continues, cours de physique théorique, tome VIII (ed. Mir) Moscou, 1969.

[12] J. Simon, Compact sets in the space $L^{p}(0, T ; B)$, Ann. Mat. Pura Appli. 146 (1987), no. 4, 65-96.

[13] A.Thiaville, Y.Nakatani, J.Miltat and Y.Susuki, Micromagnetic understanding of current driven domain wall motion in patterned nanowires, Europhys. Lett. 69 (2005), no. 6, 990996.

[14] A.Thiaville, Y.Nakatani, J.Miltat and N. Vernier, Domain wall motion by spin-polarized current: a micromagnetic study, J. Appl. Phys., Part 2, 95 (2004), no. 11, 7049-7051.

[15] A.Thiaville, J.M.Garcia and J.Miltat, Domain wall dynamics in nanowires. Journal of Magnetism and Magnetic Materials 242-245 (2002) 1061-1063.

[16] Augusto Visintin, On Landau Lifschitz equation for ferromagnetism, Japan Journal of Applied Mathematics 1 (1985), no. 2, 69-84. 